\title{
Railway Vehicle Dynamics under Degraded Adhesion Conditions: an Innovative HIL Architecture for Braking Tests on Full-Scale Roller-rigs
}

\author{
B. Allotta ${ }^{1}$, R. Conti ${ }^{1}$, E. Meli ${ }^{1}$, L. Pugi ${ }^{1}$, A. Ridolfi ${ }^{1}$ \\ ${ }^{1}$ Department of Industrial Engineering, University of Florence, Florence, Italy
}

\begin{abstract}
In this paper an innovative Hardware In the Loop (HIL) architecture to test braking on board subsystems on full-scale roller-rigs is described. The new approach permits to reproduce on the roller-rig a generic wheel-rail adhesion pattern and, in particular, degraded adhesion conditions. The presented strategy is also followed by the innovative full-scale roller-rig of the Railway Research and Approval Center of FirenzeOsmannoro (Italy); the new roller-rig has been built by Trenitalia and is owned by SIMPRO. At this initial phase of the research activity, to effectively validate the proposed approach, a complete model of the HIL system has been developed. The complete numerical model is based on the real characteristics of the components provided by Trenitalia. The results coming from the simulation model have been compared to the experimental data provided by Trenitalia and relative to on-track tests performed in Velim, Czech Republic, with a UIC-Z1 coach equipped with a fully-working WSP system. The preliminary validation performed with the HIL model highlights the good performance of the HIL strategy in reproducing on the roller-rig the complex interaction between degraded adhesion conditions and railway vehicle dynamical behaviour during the braking manoeuvre.
\end{abstract}

Keywords: Hardware In the Loop, Railway Braking, Degraded Adhesion Conditions, Roller-Rigs.

\section{Introduction}

Nowadays the longitudinal train dynamics is almost totally controlled by on board subsystems, such as Wheel Slide Protection (WSP) braking devices. The study and the development of these systems are fundamental for the vehicle safety, especially at 
high speeds and under degraded adhesion conditions. On-track tests are currently quite expensive in terms of infrastructure and vehicle management. Consequently, to reduce these costs, full-scale roller-rigs are traditionally employed to investigate the performances of braking subsystems [1] [2] [3] [4] [8]. However, in presence of degraded adhesion, the use of roller-rigs is still limited to few applications (see for example full-scale roller-rigs for the study of the wear [5], HIL systems for WSP tests [9] [25] and full-scale roller-rigs for locomotive tests [6] [9] [25]) because the large slidings between rollers and wheelsets produce wear of the rolling surfaces [28] [29]. This circumstance is very dangerous and not acceptable: the flange wear can lead to the vehicle derailment while the tread wear can produce hunting instability of the vehicle [30] [31]. Furthermore the wheel flats may generate unsafe vibrations of the vehicle on the roller-rig. Finally the wear of the rolling surfaces deeply affects the maintenance costs: the rollers have to be frequently turned or substituted.

In this work an innovative Hardware In the Loop (HIL) architecture to test braking on board subsystems on full-scale roller-rigs is presented by the authors. The new Hardware In the Loop (HIL) architecture is able to reproduce, on the roller-rig, the generic vehicle behavior on the track (especially under degraded adhesion conditions characterized by an adhesion coefficient less than 0.10) simulated by a simplified virtual train model. The HIL architecture reproduces the vehicle behavior in terms wheelsets angular velocities, torques applied on the wheelsets and tangential forces. More in detail, this goal is reached by means of an impedance simulation without having high sliding between the wheel and roller surfaces. In other words, thanks to the action of the roller motors controllers, the vehicle wheels perceive the same angular velocities, applied torques and tangential forces that they would perceive on the track, without having dangerous high sliding between the wheel and roller surfaces (since the real adhesion coefficient between rollers and wheelsets is higher than 0.30 , negligible sliding occurs between them).

The main benefits of the described approach can be summarized as follows:

- possibility to design and test the on-board subsystems for high speed trains under generic wheel-rail adhesion conditions (especially degraded adhesion conditions) on test-rigs and with low wheel and roller wear (due to negligible sliding between the contact surfaces);

- possibility to avoid expensive on track tests under normal and degraded adhesion conditions;

- possibility to avoid expensive tests on classical test-rigs under degraded adhesion conditions involving high wheel and roller wear due to large sliding between the contact surfaces;

- high accuracy thanks to the new control strategy based on the independent control of the system actuators (IPM synchronous motors).

At the same time, the following limitations are present: 
- up to now only straight tracks and braking/traction maneuvers can be analyzed;

- currently the physical characteristics of the contaminant along the track have been supposed to be uniform and constant;

- the performance of the system has to be verified and validated through test cases based on on-track experimental campaigns;

- the safety of the whole plant (due to possible system instabilities) is a very important issue and has to be accurately taken into account.

At this initial phase of the research activity, the described strategy has been completely simulated in the Matlab-Simulink environment [43] through an accurate model of the whole HIL architecture. Each model component is modelled according to the real characteristics provided by Trenitalia.

The roller-rig is designed to quickly and effectively test many different railway vehicles in many different operating conditions. Therefore the HIL architecture cannot be too complicated and require the knowledge of geometrical/physical data related to the specific vehicle (often unknown or only approximately known) and of measurements directly performed on the specific vehicle; this way, also the set-up and the management of the experimental campaigns will be much quicker and easier. This is especially true for all the three software parts of the HIL architecture (controllers, virtual train model and torque estimators) because they are thought for a real-time implementation and have to be necessarily simple.

The proposed approach has been preliminarily validated through a comparison with the experimental data provided by Trenitalia and relative to on-track tests performed on a straight railway track (in Velim, Czech Republic) with a UIC-Z1 coach equipped with a fully-working WSP system [44] [45] [46]. This initial validation carried out through the HIL model highlights the good performances of the HIL strategy in reproducing on the roller-rig the complex interaction between degraded adhesion conditions and railway vehicle dynamical behaviour during the braking manoeuvre.

Currently, the construction of the test-rig is almost completed and the whole plant will be functioning very soon. Therefore, at this phase, the preliminary validation of the HIL architecture model performed comparing the whole model to suitable experimental data coming from on track tests is very important because many parts of the simulated model will be part of the real HIL architecture (see for example the virtual train model, the controllers and the estimators). During the next step of the research activity (scheduled for this winter), the HIL architecture model will be compared to experimental data directly coming from the real HIL architecture (comprising the real fully working test-rig). This phase will be fundamental for the model validation and to draw solid conclusions about the test-rig effectiveness and efficiency. 


\section{General architecture of the HIL system}

In this chapter the architecture of the Firenze-Osmannoro HIL system is briefly described. Fig. 1 schematically shows the main parts of the architecture (both for the hardware components and the software ones). The models used to simulate all these parts will be better explained in chapter 3 .

The architecture comprises four main elements:

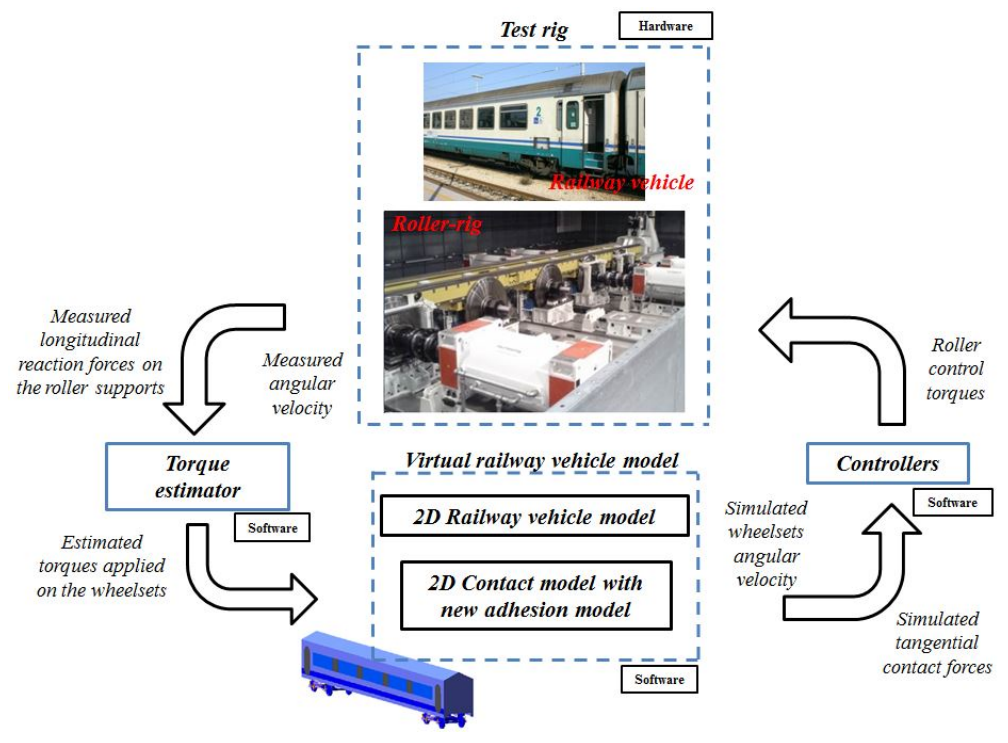

Figure 1: General architecture of the HIL system

- the test-rig (hardware), composed of two main parts: the 3D multibody models of the UIC-Z1 railway vehicle (equipped with the WSP system and the 3D wheel-roller contact model) [44] [45] and of the Firenze-Osmannoro roller-rig (with the innovative actuation system developed in collaboration with SICME and based on IPM synchronous motor with high performances) [47] [48]. The inputs of the test-rig are the roller control torques while the outputs are the longitudinal reaction forces measured on the roller supports and the measured angular velocities of the rollers;

- the virtual railway vehicle model (software), representing the model used to simulate the vehicle behaviour on the rails under different adhesion conditions [9] [25] [26] [27] and designed for a real-time implementation. This 2D multibody model simulates the longitudinal and vertical dynamical behaviour of the vehicle while an innovative $2 \mathrm{D}$ adhesion model [9] [25] permits a reproduction of the real behaviour of the adhesion coefficient during braking phases under degraded adhesion conditions.

This simplified model is used to simulate the vehicle behavior on the track under 
different adhesion conditions (especially degraded adhesion). This vehicle behavior will be reproduced on the test-rig by the motors controllers thanks to the new HIL architecture and the new control approach. In particular the new approach allows to reproduce on the test-rig the same adhesion conditions present on the track (between wheel and rail) by means of an impedance simulation without having high sliding between the wheel and roller surfaces. In other words the vehicle wheels perceive the same angular velocities, applied torques and tangential forces that they would perceive on the track without having dangerous high sliding between the wheel and roller surfaces.

The inputs are the estimated torques on the wheelsets and the outputs are the simulated wheelset angular velocities and the tangential contact forces on the wheelsets;

- the controllers (software) are all independent and reproduce on the roller-rig the same dynamical behaviour of the virtual train model (through the roller control torques) in terms of wheelset angular velocities, applied torques and, consequently, tangential forces [9] [25]. Due to the HIL system non-linearities, a sliding mode approach has been adopted for the controllers [39] [40];

- the torque estimators (software): the data measured by the sensors installed on the roller-rig are only the roller angular velocities and the longitudinal reaction forces on the roller supports. No sensors will be placed on the vehicle to speed up the set up process. Starting from these quantities, this block estimates the torques applied on the wheelsets.

\section{Modelling of the Firenze-Osmannoro HIL system}

In this section the models of the HIL system presented in the previous section (both hardware and software parts) and of all the components of the HIL architecture will be explained in detail. The main elements of the architecture are the same of Figure 1 . but, in this case, the test-rig is completely simulated both for the UIC-Z1 railway vehicle (3D vehicle model and WSP model) and for the roller-rig (roller-rig 3D model). An innovative 3D contact model especially developed by the authors for this kind of application is used [7] [21] [22]. The mechanical and electrical characteristics of the vehicle [44] [45] and of the roller-rig [47] [48] are directly provided by Trenitalia and RFI.

The flow of the data among the model parts is shown in Fig. 2. The following physical quantities are present:

- the simulated normal and tangential contact forces $N_{c s}, T_{\text {sim }}$;

- the simulated wheelset translational and rotational velocities $v_{w s}, \omega_{w s}$; 
- the original and modulated braking torques $C, C_{s}$ and the estimated one $\widehat{C}_{s}$;

- the roller control torques $u^{r / l}$;

- the real normal and tangential contact forces $\mathbf{N}_{\mathbf{c}}^{1 / \mathbf{r}}, \mathbf{T}_{\mathbf{c}}^{\mathrm{l} / \mathbf{r}}$

- the wheel and roller positions $\mathrm{G}_{\mathrm{w}}, \mathrm{G}_{\mathrm{r}}^{\mathrm{l} / \mathrm{r}}$, orientations $\Phi_{\mathrm{w}}, \boldsymbol{\Phi}_{\mathrm{r}}^{\mathrm{l} / \mathrm{r}}$, velocities $\mathbf{v}_{\mathrm{w}}$, $\mathbf{v}_{\mathbf{r}}^{1 / \mathbf{r}}$ and angular velocities $\omega_{\mathbf{w}}, \omega_{\mathbf{r}}^{1 / \mathbf{r}}$;

- the estimated wheel angular velocities $\widehat{\omega}_{w}$;

- the measured tangential contact forces $T_{m i s}^{l / r}$.

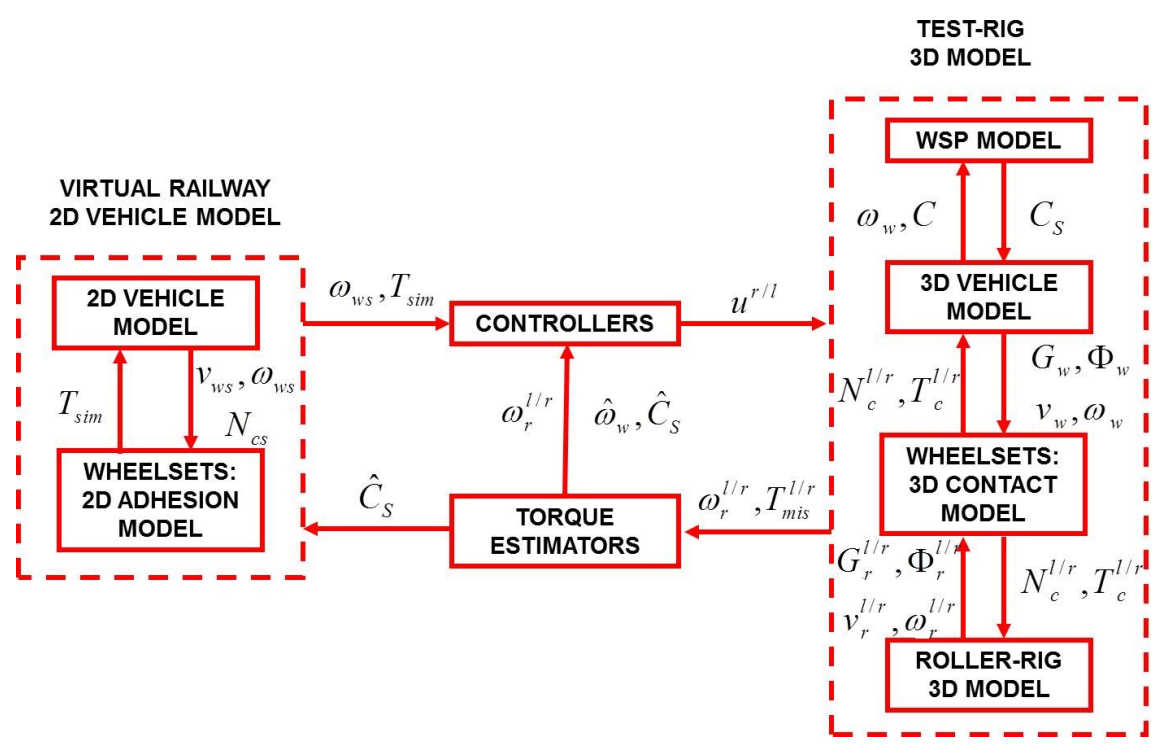

Figure 2: Interactions among the models of the various HIL architecture components

\subsection{The Test-rig Model}

The inputs of the whole test-rig model are the 8 roller control torques $u^{l}, u^{r}$ (left and right) evaluated by the controllers to reproduce on the test-rig the same dynamical behaviour of the virtual railway model. The outputs are the 8 roller angular velocities $\omega_{r}^{l}, \omega_{r}^{r}$ and the longitudinal reaction forces $T_{m i s}^{l}, T_{m i s}^{r}$ measured on the roller supports. The test-rig model is composed of four parts (Fig. 2):

\subsubsection{The Vehicle Model}

The considered railway vehicle is the UIC-Z1 vehicle (illustrated in Fig. 3); its geometrical and physical characteristics are provided by Trenitalia S.p.A. [44].

The 3D multibody vehicle model is composed of one carbody, two bogie frames, eight 


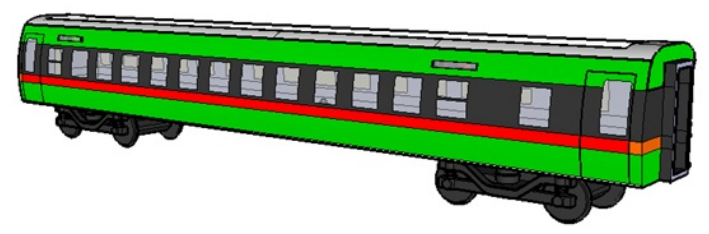

Figure 3: Multibody model of the UIC-Z1 railway vehicle

axleboxes and four wheelsets (the wheel profile is the ORE S1002). The primary suspension, including springs, dampers and axlebox bushings, connects the bogie frame to the four axleboxes while the secondary suspension, including springs, dampers, lateral bump-stops, anti-roll bar and traction rod, connects the carbody to the bogie frames (see Fig. 4). In Tab. 1 the main properties of the railway vehicle are given. The multibody vehicle model takes into account all the degrees of freedom (DOFs) of

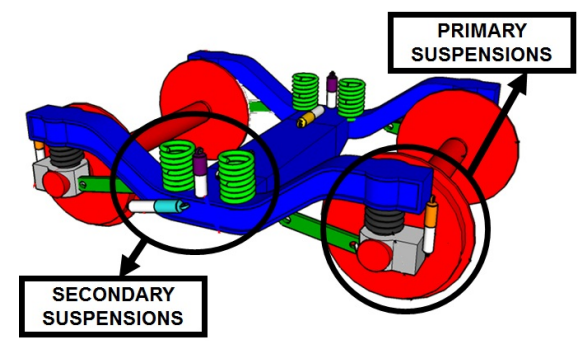

Figure 4: Primary and secondary suspensions

Table 1: Main characteristics of the railway vehicle

\begin{tabular}{lcc}
\hline Parameter & Units & Value \\
\hline Total mass & {$[\mathrm{kg}]$} & $\approx 43000$ \\
Wheel arrangement & - & $2-2$ \\
Bogie wheelbase & {$[\mathrm{m}]$} & 2.56 \\
Bogie distance & {$[\mathrm{m}]$} & 19 \\
Wheel diameter & {$[\mathrm{m}]$} & 0.89 \\
Primary suspended masses own frequency & {$[\mathrm{Hz}]$} & $\approx 4.5$ \\
Secondary suspended masses (carbody) own frequency & {$[\mathrm{Hz}]$} & $\approx 0.8$ \\
\hline
\end{tabular}

the system bodies (one carbody, two bogie frames, eight axleboxes, and four wheelsets). Considering the kinematic constraints that link the axleboxes and the wheelsets (cylindrical 1DOF joints) and without including the wheel-rail contacts, the whole system has 50 DOFs. The main inertial properties of the bodies are summarized in Tab. 2. [44] Both the primary suspension (springs, dampers and axlebox bushings) and the secondary suspension (springs, dampers, lateral bump-stops, anti-roll bar and traction rod) have been modelled through $3 \mathrm{D}$ visco-elastic force elements able to describe all the main non-linearities of the system (see Fig. 4). In Tab. 3 the characteristics of the main linear elastic force elements of both the suspension stages are reported. [44] The non-linear elastic force elements have been modelled through non-linear functions that correlate the displacements and the relative velocities of the force elements 
Table 2: Inertial properties of the rigid bodies

\begin{tabular}{|c|c|c|c|c|}
\hline Body & Mass $[\mathrm{kg}]$ & $\begin{array}{ll}\mathrm{I}_{x x} & {\left[\mathrm{kgm}^{2}\right]}\end{array}$ & $\mathrm{I}_{y y} \quad\left[\mathrm{kgm}^{2}\right]$ & $\mathrm{I}_{z z} \quad\left[\mathrm{kgm}^{2}\right]$ \\
\hline Carbody & $\approx 29000$ & 76400 & 1494400 & 1467160 \\
\hline Bogie & $\approx 3000$ & 2400 & 1900 & 4000 \\
\hline Wheelset & $\approx 1300$ & 800 & 160 & 800 \\
\hline Axlebox & $\approx 200$ & 3 & 12 & 12 \\
\hline
\end{tabular}

connection points to the elastic and damping forces exchanged by the bodies.

The inputs of the model are the 4 wheelset torques $C_{s}$ modulated by the on board

Table 3: Main linear elastic characteristics of the two stage suspensions

\begin{tabular}{lcccccc}
\hline Element & $\begin{array}{c}\text { Transl. } \\
\text { Stiff. x } \\
{[\mathrm{N} / \mathrm{m}]}\end{array}$ & $\begin{array}{c}\text { Transl. } \\
\text { Stiff. y } \\
{[\mathrm{N} / \mathrm{m}]}\end{array}$ & $\begin{array}{c}\text { Transl. } \\
\text { Stiff. } z \\
{[\mathrm{~N} / \mathrm{m}]}\end{array}$ & $\begin{array}{c}\text { Rotat. } \\
\text { Stiff. x } \\
{[\mathrm{Nm} / \mathrm{rad}]}\end{array}$ & $\begin{array}{c}\text { Rotat. } \\
\text { Stiff. y } \\
{[\mathrm{Nm} / \mathrm{rad}]}\end{array}$ & $\begin{array}{c}\text { Rotat. } \\
\text { Stiff. z } \\
{[\mathrm{Nm} / \mathrm{rad}]}\end{array}$ \\
\hline $\begin{array}{l}\text { Springs of the } \\
\text { primary suspension }\end{array}$ & 844000 & 844000 & 790000 & 10700 & 10700 & 0 \\
$\begin{array}{l}\text { Springs of the } \\
\text { secondary suspension }\end{array}$ & 124000 & 124000 & 340000 & 0 & 0 & 0 \\
$\begin{array}{l}\text { Axlebox bushing } \\
\text { Anti-roll bar }\end{array}$ & $4 \cdot 10^{7}$ & $6.5 \cdot 10^{6}$ & $4 \cdot 10^{7}$ & 45000 & 9700 & 45000 \\
\hline
\end{tabular}

WSP and the contact forces calculated by the contact model, while the outputs are the kinematic wheelset variables transmitted to the contact model, the 4 original torques $C$ (without the on board WSP modulation) and the 4 wheelset angular velocities $\omega_{w}$. These last two outputs are not accessible by the HIL system.

\subsubsection{The Wheel Slide Protection System Model}

The WSP device installed on the UIC-Z1 coach [45] [9] [25] allows the control of the torques applied to the wheelsets, to prevent macro-sliding during the braking phase.

In Figure 5 the logical scheme and an image of the WSP device are shown. The inputs are the braking torques $C$ and the wheelset velocities $\omega_{w}$, while the outputs are the modulated braking torques $C_{s}$. The WSP system working principle can be divided into three different tasks: the evaluation of the reference vehicle velocity $V_{\text {ref }}$ and acceleration $a_{r e f}$ based on the wheelset angular velocities $\omega_{w}$ and accelerations $\dot{\omega}_{w}$; the computation of the logical sliding state state $_{W S P}$ (equal to 1 if sliding occurs and 0 otherwise)and the consequent torque modulation, through a speed and an accelerometric criterion and by means of a suitable logical table [9] [25]; the periodic braking release to bring back the perceived adhesion coefficient to the original value (often used when degraded adhesion conditions are very persistent and the WSP logic tends to drift).

The system tries to keep the wheelset peripheral speed $r_{w} \omega_{w}$ in an optimal interval that is variable according to the reference speed value $V_{\text {ref }}$ : this interval is defined by two different speed levels $V_{1}$ and $V_{2}$, which are functions of $V_{\text {ref }}$. The peripheral speed of each wheelset $r_{w} \omega_{w}$ is compared to $V_{1}$ and $V_{2}$ causing EVR and EVS valve actions on braking pressure. The speed criterion (which directly controls the wheelset creepages, similarly to a proportional controller) has some limits: the control dead-zone between 


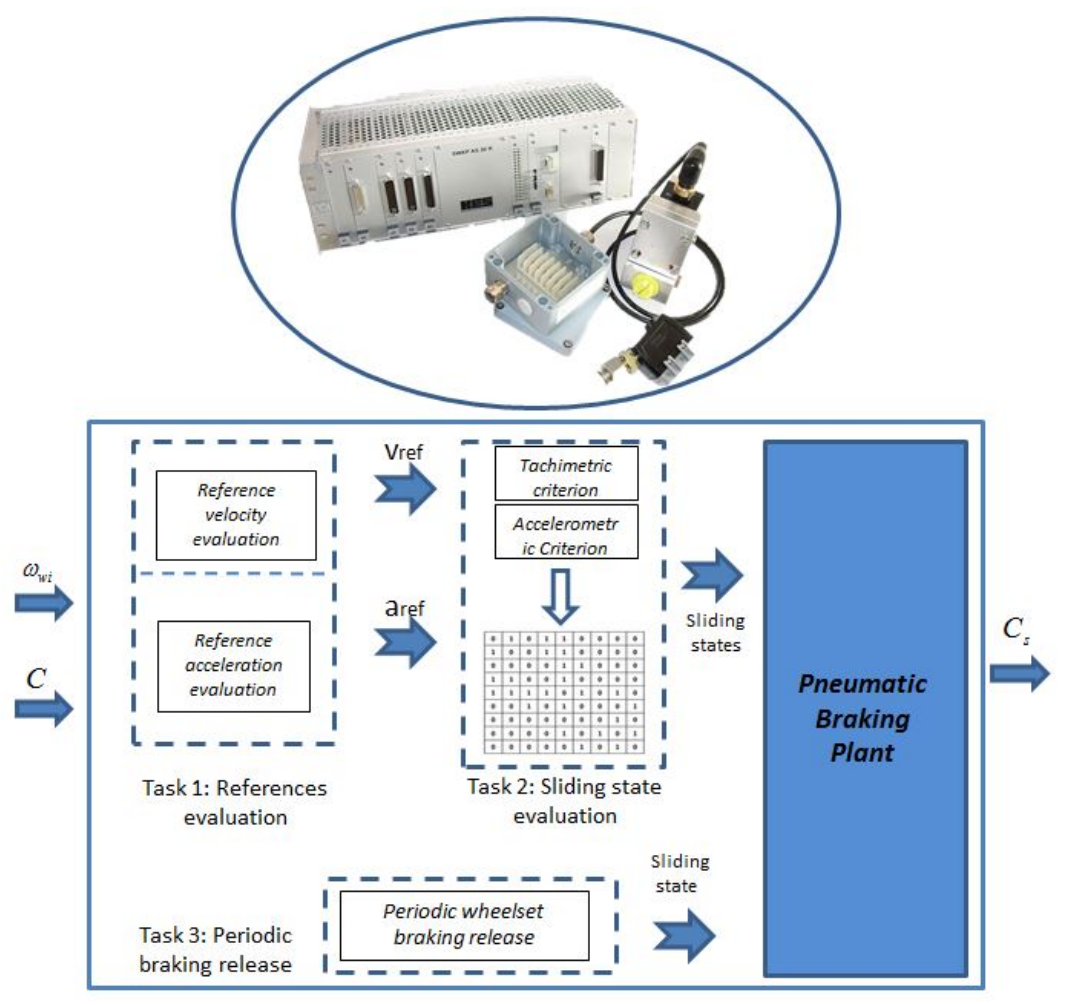

Figure 5: WSP device and its logical scheme

$V_{1}$ and $V_{2}$ and the slow dynamic response of the braking cylinder may cause a not optimal slip control. In order to increase WSP slip control performances within the speed interval defined by $V_{1}$ and $V_{2}$, an acceleration criterion is added to the speed one: this acceleration criterion is based on the reference acceleration $a_{r e f}$ and on the wheelset peripheral acceleration $r_{w} \dot{\omega}_{w}$ and, similarly to a derivative controller, causes EVR and EVS valve actions on braking pressure.

\subsubsection{The Roller-rig Model}

The 3D multibody model of the roller-rig (see Fig. 6 and Tab. 4) consists of 8 indipendent rollers with a particular roller profile able to exactly reproduce the UIC60 rail pattern with different laying angles $\alpha_{p}$ (the inclination of the rail profile with respect to the rail plane) [47]. Due to the nature of the system, different kind of instabilities may arise, especially concerning the dynamics of the wheelsets on the rollers and, in the worst case, may also lead to the fallen from the roller-rig. To avoid these dangerous situations, a fixed and a mobile constrain (to hold different kind of vehicles both in their front side and in their rear one) are installed. In order to simulate these real hooks, the front and the rear side of the vehicle are constrained on the rollers by 3D force elements with linear stiffness and damping; these elements can simulate different types of constrains. In the standard situation the translations along the xyz axes and the rotations around the yz directions are not permitted (5 DOFs constrained). The 
inputs of the test-rig model are the 8 torques $u^{l}, u^{r}$ evaluated by the controllers and the contact forces calculated by the contact model; the outputs are the roller angular velocities $\omega_{r}^{l}$, $\omega_{r}^{r}$, the longitudinal reaction forces $T_{m i s}^{l}, T_{m i s}^{r}$ measured on the roller supports and the kinematic wheelset variables transmitted to the contact model.

The roller-rig actuation system consists of 8 synchronous motors, especially designed

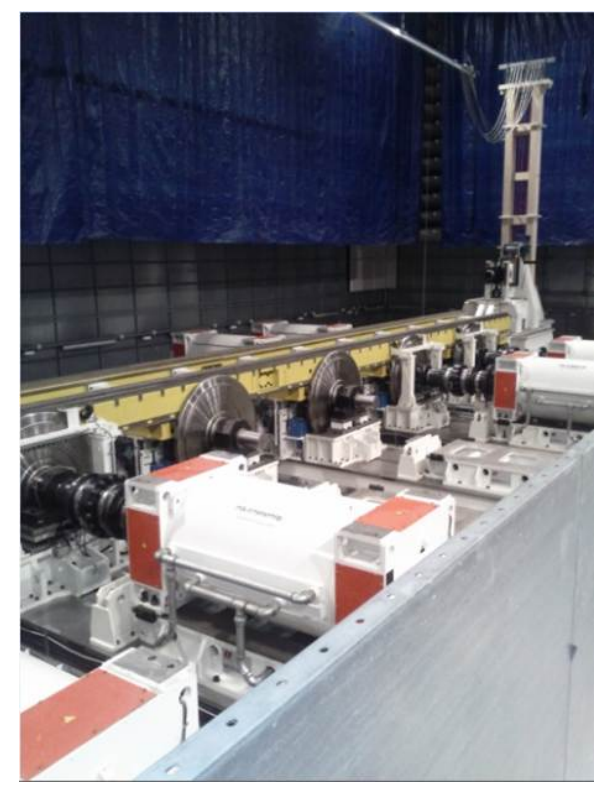

Table 4: Main characteristics of the roller-rig system and of the wheelsets

\begin{tabular}{lcc}
\hline Parameter & Units & Value \\
\hline Roller radius $r_{r}$ & $\mathrm{~m}$ & 0.725 \\
Roller mass $m_{r}$ & $\mathrm{~kg}$ & 2980 \\
Roller inertia $J_{r}$ & $\mathrm{kgm}^{2}$ & 705 \\
Wheelset radius $r_{w}$ & $\mathrm{~m}$ & 0.445 \\
Wheelset mass $m_{w}$ & $\mathrm{~kg}$ & 1300 \\
Wheelset inertia $J_{w}$ & $\mathrm{kgm}^{2}$ & 160 \\
\hline
\end{tabular}

Figure 6: The right side of rollerrig system with the synchronous motors and the rollers placed in the semi-anechoic room of the Research and Approval Center of FirenzeOsmannoro

and developed in cooperation with SICME [48] for this kind of application. The HIL architecture includes a direct-drive connection between the roller and the electrical machine. The synchronous motors have high efficiency associated with high torque density and flux weakening capability. Furthermore, to reach the dynamical and robustness performances required by the railway full-scale roller-rig, the motors are designed with a multilayer-rotor characterized by a high saliency ratio $\xi$ and Interior Permanent Magnets (IPM). The IPM motors are controlled in real-time through vector control techniques; more particularly the vector control is a torque-controlled drive system in which the controller follows a desired torque [48] [32] [33] [34] [35].

The main sensors installed on the roller-rig (see Fig. 7) are the absolute encoders and the 3-axial load cells on the roller supports. These sensors are employed both in the torque estimators and in the controllers and measure, respectively, the roller angular velocities $\omega_{r}^{l}, \omega_{r}^{r}$ and the longitudinal reaction forces $T_{m i s}^{l}, T_{m i s}^{r}$ on the roller supports. The sensor characteristics are reported in Tab. 5] [48].

More particularly, the 3-axial load cell is used to measure the force along the xyz di- 


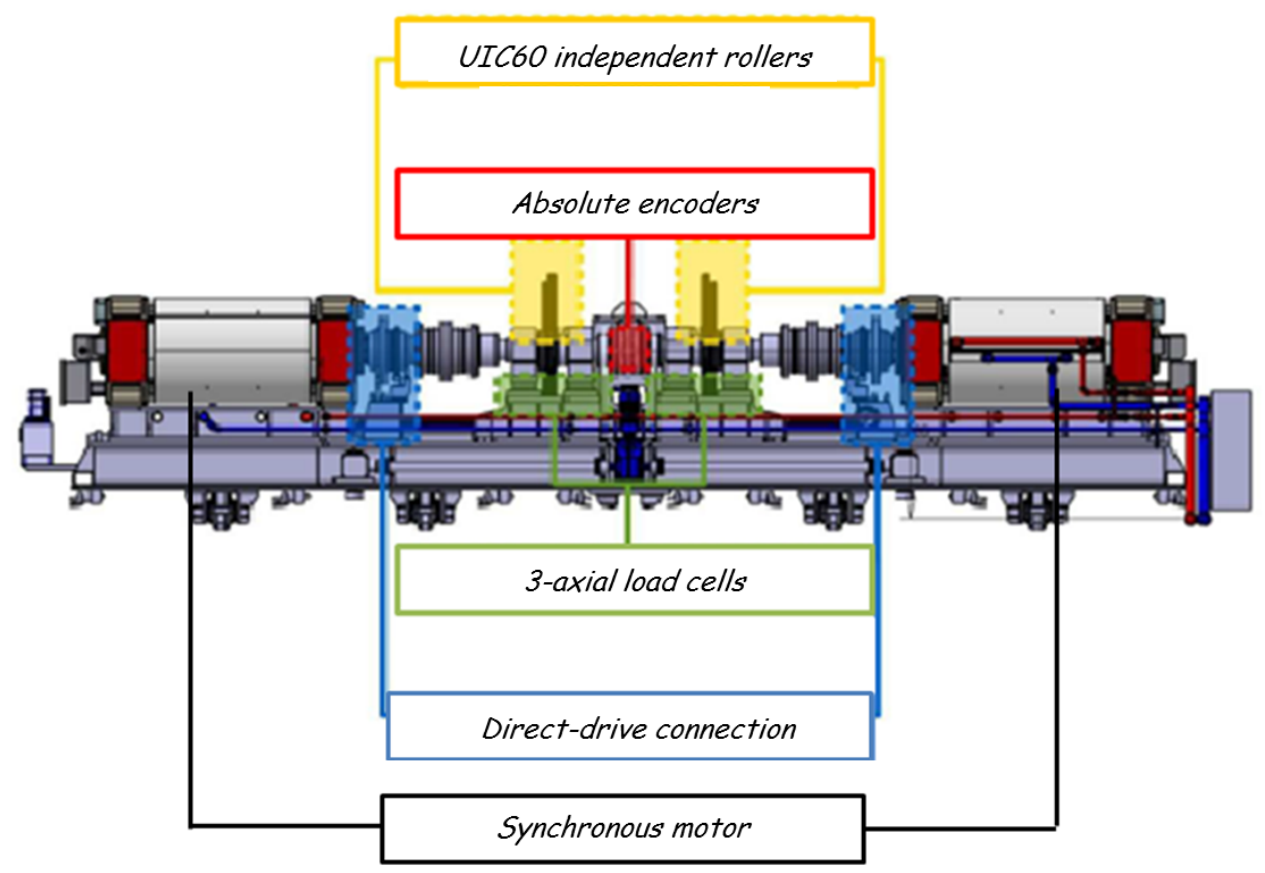

Figure 7: Main roller-rig sensors

Table 5: Roller-rig sensor characteristics

\begin{tabular}{lllll}
\hline Sensor & Range & Resolution & Sensitivity & Passband \\
\hline Absolute encoder & $2 \pi \mathrm{rad}$ & $7 * 10^{-5} \mathrm{rad}$ & $\pm 0.01 \%$ full scale & - \\
3-axial load cells & $\pm 200 \mathrm{kN}$ & $7 \mathrm{~N}$ & $\pm 0.1 \%$ full scale & $0.1 \mathrm{kHz}$ \\
\hline
\end{tabular}

rections through several strain gauges that evaluate the deformation measuring the resistance modification with a Wheatstone bridge. In the CDSO HIL architecture, these sensors are necessary to estimate the tangential components of the contact forces at the roller-wheel interface; the 3-axial load cell are installed both on the right and the left side of the roller. As regards the 3-axial load cell model, a low-pass filter is used with a cut-off frequency based on the real passband; in addition, a variable offset is defined to simulate the sensitivity of the sensor (see Table 5).

\subsubsection{The Wheel-roller Contact Model}

The $3 \mathrm{D}$ contact model evaluates the contact forces $\mathbf{N}_{\mathbf{c}}^{1 / \mathbf{r}}, \mathbf{T}_{\mathbf{c}}^{\mathrm{l} / \mathbf{r}}$ for all the 8 wheel-roller pairs starting from the kinematic variables of the wheelsets and of the rollers: their positions $\mathrm{G}_{\mathrm{w}}, \mathrm{G}_{\mathrm{r}}^{\mathrm{l} / \mathrm{r}}$, orientations $\Phi_{\mathrm{w}}, \Phi_{\mathrm{r}}^{\mathrm{l} / \mathrm{r}}$, velocities $\mathrm{v}_{\mathrm{w}}, \mathrm{v}_{\mathrm{r}}^{1 / \mathrm{r}}$ and angular velocities $\omega_{\mathbf{w}}, \omega_{\mathbf{r}}^{1 / \mathbf{r}}$ (see Fig. 8).

The contact model comprises three different steps. Firstly, all the contact points $\mathbf{P}_{\mathbf{c}}^{\mathbf{l} / \mathbf{r}}$ of each wheel-roller pair are detected. Some innovative procedures have been recently developed by the authors [7] [21] [22]; the new algorithms are based on the reduction of the algebraic contact problem dimension through exact analytical 


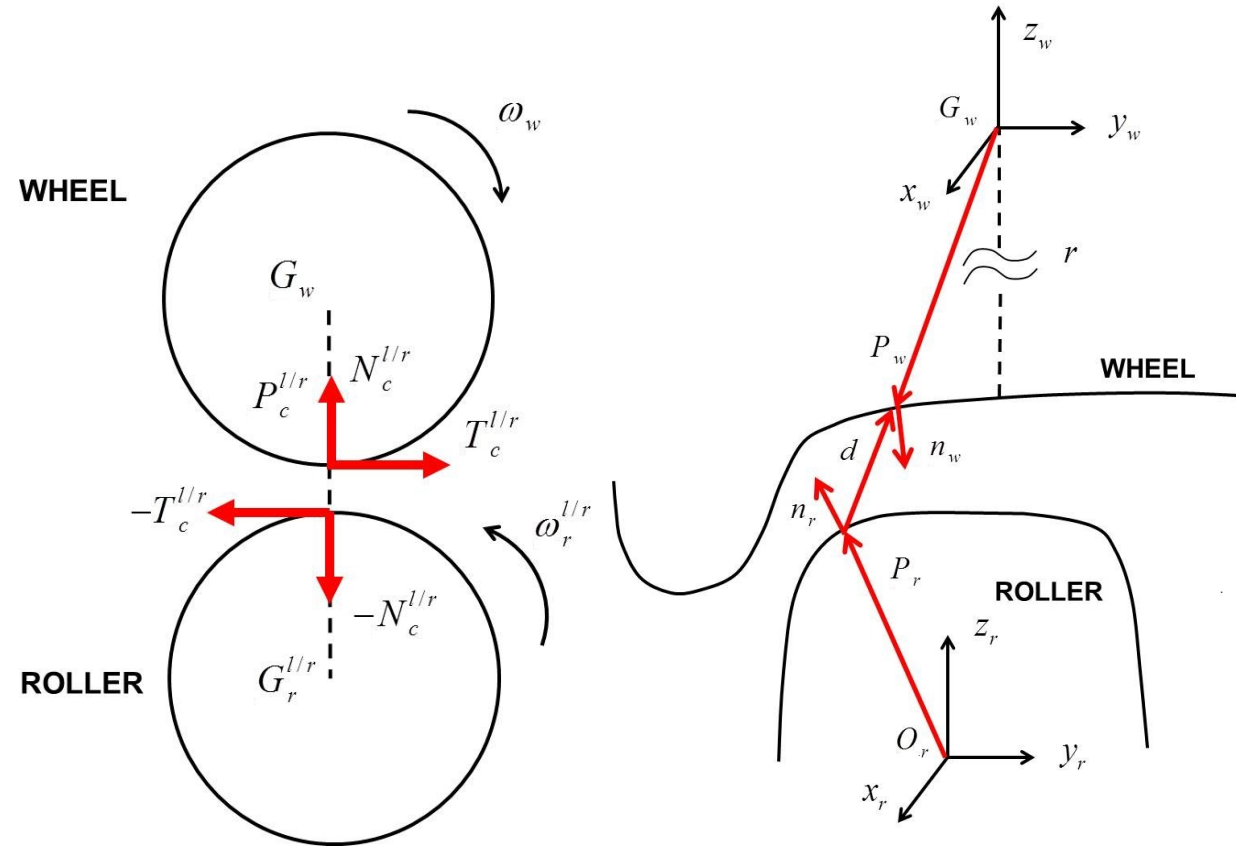

Figure 8: Wheel-roller contact Figure 9: Wheel-roller contact model point detection

techniques [23]. Secondly, the normal contact problem is solved through the Hertz theory [10] to evaluate the normal contact forces $\mathbf{N}_{\mathbf{c}}^{1 / \mathbf{r}}$. Finally, the solution of the tangential contact problem is performed by means of the Kalker-Polach theory [10] [11] [12] [13] [14] [15] [16] to compute the tangential contact forces $\mathbf{T}_{\mathbf{c}}^{\mathbf{l} \mathbf{r}}$. The contact model guarantees high accuracy and numerical efficiency; this way, the model can be implemented directly online inside the whole test-rig model.

The main characteristics of the innovative procedure for the contact points detection can be summarized as follows:

- it is a fully 3D algorithm that takes into account all the six relative DOFs between wheel and roller;

- it is able to support generic wheel and roller profiles (in this specific case the ORE S1002 for the wheels and the UIC60 with different laying angles alpha for the rollers);

- it assures a general and accurate treatment of the multiple contact without introducing simplifying assumptions on the problem geometry and kinematics and limits on the number of contact points detected; the accuracy of the 3D contact model used inside the test-rig have been accurately investigated in previous works of the authors both for the wheel-rail and for the wheel-roller case [7] [21] [22] [23]; 
- it assures high numerical efficiency making possible the online implementation within the commercial multibody software without discrete Look-up Tables [7] [21] [22] [23] [43].

The contact point position can be evaluated imposing the following parallelism conditions [41]:

$$
\begin{gathered}
\mathbf{n}_{r}^{r}\left(\mathbf{P}_{r}^{r}\right) \times \mathbf{n}_{w}^{r}\left(\mathbf{P}_{w}^{r}\right)=\mathbf{n}_{r}^{r}\left(\mathbf{P}_{r}^{r}\right) \times R_{w}^{r} \mathbf{n}_{w}^{w}\left(\mathbf{P}_{w}^{w}\right)=\mathbf{0} \\
\mathbf{n}_{r}^{r}\left(\mathbf{P}_{r}^{r}\right) \times \mathbf{d}^{r}=\mathbf{0}
\end{gathered}
$$

where

$$
\begin{gathered}
\mathbf{P}_{w}^{w}\left(x_{w}, y_{w}\right)=\left(\begin{array}{lll}
x_{w} & y_{w} & -\sqrt{w\left(y_{w}\right)^{2}-x_{w}^{2}}
\end{array}\right)^{T} \\
\mathbf{P}_{r}^{r}\left(x_{r}, y_{r}\right)=\left(\begin{array}{lll}
x_{r} & y_{r} & +\sqrt{r\left(y_{r}\right)^{2}-x_{r}^{2}}
\end{array}\right)^{T}
\end{gathered}
$$

are the positions of the generic points on the wheel surface and on the roller surface (expressed in the reference systems $G_{w} x_{w} y_{w} z_{w}$ and $\left.O_{r} x_{r} y_{r} z_{r}\right), w\left(y_{w}\right)$ and $r\left(y_{r}\right)$ are the wheel and roller profiles (supposed to be known), $\mathbf{n}_{w}^{w}$ and $\mathbf{n}_{r}^{r}$ are the outgoing normal unit vectors to the wheel and roller surfaces (in the reference systems $G_{w} x_{w} y_{w} z_{w}$ and $\left.O_{r} x_{r} y_{r} z_{r}\right), R_{w}^{r}$ is the rotation matrix that links the reference system $G_{w} x_{w} y_{w} z_{w}$ to the reference system $O_{r} x_{r} y_{r} z_{r}$ and $\mathbf{d}^{r}$ is the distance vector between two generic points on the wheel surface and on the roller surface (both referred to the reference system $\left.O_{r} x_{r} y_{r} z_{r}\right): \mathbf{d}^{r}\left(x_{w}, y_{w}, x_{r}, y_{r}\right)=\mathbf{P}_{w}^{r}\left(x_{w}, y_{w}\right)-\mathbf{P}_{r}^{r}\left(x_{r}, y_{r}\right)$ in which $\mathbf{P}_{w}^{r}=\mathbf{G}_{w}^{r}+R_{w}^{r} \mathbf{P}_{w}^{w}\left(x_{w}, y_{w}\right)$ is the position of the generic point of the wheel surface expressed in the reference system $O_{r} x_{r} y_{r} z_{r}$ (see Fig. 9).

The first condition of the system (1) imposes the parallelism between the normal unit vectors, while the second one requires the parallelism between the normal unit vector to the roller surface and the distance vector. The system (1) consists of six non-linear equations in the unknowns $\left(x_{w}, y_{w}, x_{r}, y_{r}\right)$ (only four equations are independent and therefore the problem is 4D). However it is possible to exactly express three of the four variables (in this case $\left.\left(x_{w}, x_{r}, y_{r}\right)\right)$ as a function of $y_{w}$, reducing the original 4D problem to a single $1 \mathrm{D}$ scalar equation in the variable $y_{w}[$ [7] [21] [22] [23]:

$$
F\left(y_{w}\right)=0 \text {. }
$$

At this point the simple scalar Eq. (3) can be easily solved through appropriate numerical algorithms. [37] Finally, once obtained the generic solution $y_{w c}$ of Eq. (3), the complete solution $\left(x_{w c}, y_{w c}, x_{r c}, y_{r c}\right)$ of the system (1) and consequently the contact points $\mathbf{P}_{w c}^{r}=\mathbf{P}_{w}^{r}\left(x_{w c}, y_{w c}\right)$ and $\mathbf{P}_{r c}^{r}=\mathbf{P}_{r}^{r}\left(x_{r c}, y_{r c}\right)$ can be found by substitution.

\subsection{The Virtual Railway Vehicle Model}

The virtual railway vehicle model simulates the dynamical behaviour of the railway vehicle during a braking phase under degraded adhesion conditions. The model, designed for a real-time implementation, is composed of two parts: the 2D vehicle model 
and the $2 \mathrm{D}$ adhesion model. The inputs are the 4 estimated torques $\widehat{C}_{s}$ to be applied to the wheelsets while the outputs are the 4 simulated tangential contact forces $T_{\text {sim }}$ and the 4 simulated wheel angular velocities $\omega_{w s}$.

\subsubsection{The Virtual Vehicle Model}

The 2D model of the considered railway vehicle (UIC-Z1 coach) is a simplified 2D multibody model of the longitudinal train dynamical behaviour (only 3 DOFs for each body are taken into account: longitudinal and vertical translations and pitch rotation) [44]. The model (see Fig. 10) consists of a carbody, two bogies and four wheelsets, held by the primary and secondary suspensions. Starting from the estimated torques $\widehat{C}_{s}$, the model evaluates the kinematic variables of the 4 wheelsets $v_{w s}, \omega_{w s}$ and the 4 normal contact forces $N_{c s}$ to be passed to the adhesion model and receives the 4 tangential contact forces $T_{\text {sim }}$.

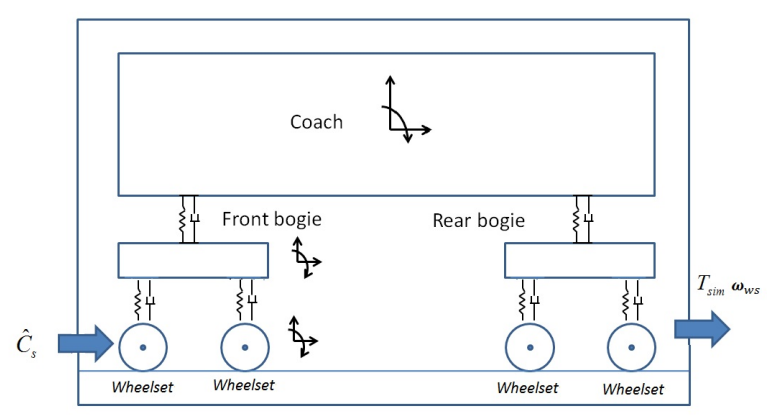

Figure 10: The virtual railway vehicle model

\subsubsection{The Adhesion Model}

The 2D adhesion model has been especially developed to describe degraded adhesion conditions [9] [25] [17] [18] [19] [20] and calculates, for all the 4 wheelset-rail pairs, the tangential contact forces $T_{\text {sim }}$ starting from the wheelset kinematic variables $v_{w s}$, $\omega_{w s}$ and the normal contact forces $N_{c s}$ (see Fig. 11p.

The main phenomena characterising the degraded adhesion are the large sliding occurring at the contact interface and, consequently, the high energy dissipation. Such a dissipation causes a cleaning effect on the contact surfaces and finally an adhesion recovery due to the removal of the external contaminants. In other words, the work of the friction forces provokes the cleaning of the contact surfaces removing the third-body between wheel and rail (oxides,soil,wear debris,water,etc.). This cleaning effect may also bring back the adhesion coefficient to values typical of dry contact conditions. More particularly, the following qualitative phases can be identified: 

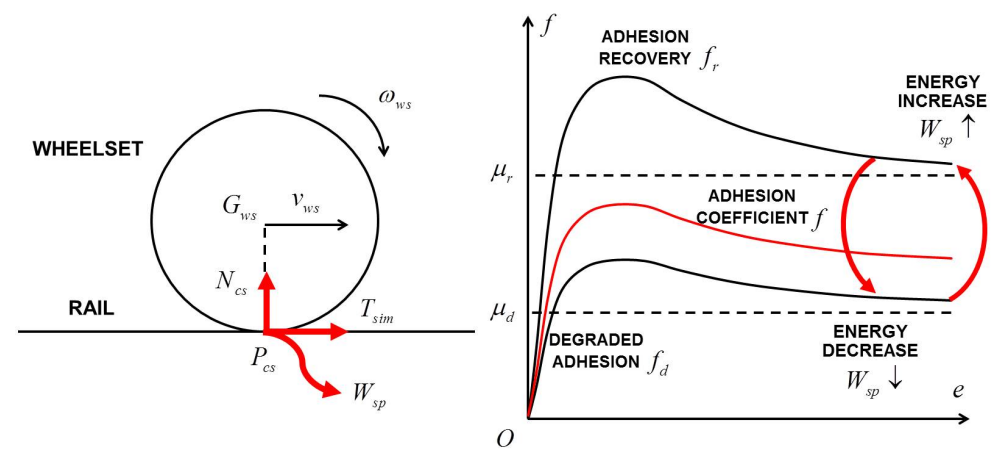

Figure 11: The adhesion model

- when the specific dissipated energy $W_{s p}$ is low the cleaning effect is almost absent, the contaminant level $h$ does not change and the adhesion coefficient $f$ is equal to its original value $f_{d}$ in degraded adhesion conditions $f_{d}$;

- as the energy $W_{s p}$ increases, the cleaning effect increases too, the contaminant level $h$ becomes thinner and the adhesion coefficient $f$ raises;

- in the end, for large values of $W_{s p}$, all the contaminant is removed ( $h$ is null) and the adhesion coefficient $f$ reaches its maximum value $f_{r}$; the adhesion recovery due to the removal of external contaminants is now completed;

- at the same time if the energy dissipation begins to decrease, due for example to a lower sliding, the reverse process occurs (see Fig. 11). Finally it has to be noted that the values of the adhesion coefficients $f_{d}$ and $f_{r}$ can never be equal (naturally being equal the values of the other parameters).

Since the contaminant level $h$ and its characteristics are usually totally unknown, it is usefull trying to experimentally correlate the adhesion coefficient $f$ directly with the specific dissipated energy $W_{s p}$ :

$$
W_{s p}=T_{s i m} e=f N_{c s} e \quad f=\frac{T_{s i m}}{N_{c s}}
$$

where the creepage $e$ is defined as

$$
e=\frac{s}{v_{w s}}=\frac{v_{w s}-r_{w} \omega_{w s}}{v_{w s}}
$$

$s$ is the sliding and $r_{w}$ is the wheel radius. This way the specific dissipated energy $W_{s p}$ can also be interpreted as the energy dissipated at the contact for unit of distance travelled by the railway vehicle.

To reproduce the qualitative trend previously described and to allow the adhesion coefficient to vary between the extreme values $f_{d}$ and $f_{r}$, the following expression for $f$ is proposed:

$$
f=\left[1-\lambda\left(W_{s p}\right)\right] f_{d}+\lambda\left(W_{s p}\right) f_{r}
$$


where $\lambda\left(W_{s p}\right)$ is an unknown transition function between degraded adhesion and adhesion recovery while the adhesion levels $f_{d}, f_{r}$ can be evaluated according to [11] [12] [13] [14] [15] [16] as a function of $e, N_{c s}$ and the track friction coefficients $\mu_{d}, \mu_{r}$ (corresponding to degraded adhesion and full adhesion recovery, respectively). The function $\lambda\left(W_{s p}\right)$ has to be positive and monotonous increasing; moreover the following boundary conditions are supposed to be verified: $\lambda(0)=0$ and $\lambda(+\infty)=1$.

This way, the authors suppose that the transition between degraded adhesion and adhesion recovery only depends on $W_{s p}$. This hypothesis is obviously only an approximation but, as it will be clearer in the next chapters, it well describes the adhesion behaviour. Initially, to catch the physical essence of the problem without introducing a large number of unmanageable and unmeasurable parameters, the authors have chosen the following simple expression for $\lambda\left(W_{s p}\right)$ :

$$
\lambda\left(W_{s p}\right)=1-e^{-\tau W_{s p}}
$$

where $\tau$ is now the only unknown parameter to be tuned on the base of the experimental data (in this case $\tau=1.9 * 10^{-4} \mathrm{~m} / \mathrm{J}$ ) [25] [24] [46] [9] [7] [36].

More in detail, starting from the experimental transition functions $\lambda_{j k}^{s p}\left(W_{s p j k}^{s p}\right)$ corresponding to the $j-t h$ vehicle wheel during the $k-t h$ experimental test, the parameter $\tau$ within $\lambda\left(W_{s p}\right)$ has been calculated through a Non-linear Least Square Optimization (NLSO) by minimizing the following error function:

$$
g(\tau)=\sum_{k=1}^{N_{e}} \sum_{i=1}^{N_{t}} \sum_{j=1}^{N_{w}}\left[\lambda_{j k}^{s p}\left(W_{j k}^{s p}\left(t_{i}\right)\right)-\lambda\left(W_{j k}^{s p}\left(t_{i}\right)\right)\right]^{2}
$$

where $N_{w}$ is the wheel number, $N_{e}$ is the number of experimental tests and $N_{t}$ is the measured sample number.

The function $\lambda\left(W_{s p}\right)$ has not a physical foundation but it is a transition function between the two main adhesion levels $f_{d}$ and $f_{r}$. At the current step, this transition function has been chosen because it represents a very good compromise among accuracy, simplicity and numerical efficiency (it is very easy to be calculated and is characterized by only one unknown parameter to be tuned and physically interpreted). Despite its simplicity, the chosen transition function well satisfies the required boundary conditions and well reproduce the general trend of the physical phenomenon. Moreover such function is quite robust and universal because it modestly depends on the specific operating conditions (see the validation of the adhesion model described in [25] [24] taking in account different experimental tests). During this initial phase of the research activity, also polynomial and look-up table based transition functions have been considered. Such functions provide analogous results in terms of accuracy but they are quite more complicated to be tuned and interpreted (many unknown parameters are present) and turn out to be much heavier from a computational viewpoint. Furthermore these functions are also more sensitive to the operating conditions.

In this research activity the two main adhesion coefficients $f_{d}$ and $f_{r}$ (degraded adhesion and adhesion recovery) have been calculated according to Polach: [11] [12] [10] 
[13] [14] [15] [16]

$$
\begin{aligned}
& f_{d}=\frac{2 \mu_{d}}{\pi}\left[\frac{k_{a d} \varepsilon_{d}}{1+\left(k_{a d} \varepsilon_{d}\right)^{2}}+\operatorname{arctg}\left(k_{s d} \varepsilon_{d}\right)\right] \\
& f_{r}=\frac{2 \mu_{r}}{\pi}\left[\frac{k_{a r} \varepsilon_{r}}{1+\left(k_{a r} \varepsilon_{r}\right)^{2}}+\operatorname{arctg}\left(k_{s r} \varepsilon_{r}\right)\right]
\end{aligned}
$$

where

$$
\varepsilon_{d}=\frac{2}{3} \frac{C \pi a^{2} b}{\mu_{d} N_{c}} e \quad \varepsilon_{r}=\frac{2}{3} \frac{C \pi a^{2} b}{\mu_{r} N_{c}} e .
$$

The quantities $k_{a d}, k_{s d}$ and $k_{a r}, k_{s r}$ are the Polach reduction factors (for degraded adhesion and adhesion recovery respectively) and $\mu_{d}, \mu_{r}$ are the friction coefficient defined as follows

$$
\mu_{d}=\left(\frac{\mu_{c d}}{A_{d}}-\mu_{c d}\right) e^{-\gamma_{d} s}+\mu_{c d} \quad \mu_{r}=\left(\frac{\mu_{c r}}{A_{r}}-\mu_{c r}\right) e^{-\gamma_{r} s}+\mu_{c r}
$$

in which $\mu_{c d}, \mu_{c r}$ are the kinetic friction coefficients, $A_{d}, A_{r}$ are the ratios between the kinetic friction coefficients and the static ones and $\gamma_{d}, \gamma_{d}$ are the friction decrease rates. The Polach approach (see Eq. (9)) has been followed since it permits to describe the decrease of the adhesion coefficient with increasing creepage and to better fit the experimental data (see Fig. 11).

Finally it has to be noticed that the semi-axes $a$ and $b$ of the contact patch (see Eq. (10)) depend only on the material properties, the contact point position $P_{c}$ on wheel and rail (through the curvatures of the contact surfaces in the contact point) and the normal force $N_{c}$, while the contact shear stiffness $C\left(\mathrm{~N} / \mathrm{m}^{3}\right)$ is a function only of material properties, the contact patch semi-axes $a$ and $b$ and the creepages. More particularly, the following relation holds: [10]

$$
C=\frac{3 G}{8 a} \sqrt{\left(c_{11} \frac{e_{x}}{e}\right)^{2}+\left(c_{22} \frac{e_{y}}{e}\right)^{2}}
$$

where $c_{11}=c_{11}(\sigma, a / b)$ and $c_{22}=c_{22}(\sigma, a / b)$ are the Kalker coefficients.

In the end, the desired values of the adhesion coefficient $f$ and of the tangential contact force $T_{\text {sim }}=f N_{c s}$ can be evaluated by solving the non-linear algebraic Eq. (6) in which the explicit expression of $W_{s p}$ has been inserted (see Eq. (4p):

$$
f=\Im(f, t)
$$

where $\Im$ indicates the generic functional dependence. Due to the simplicity of the transition function $\lambda\left(W_{s p}\right)$, the solution can be easily obtained through standard nonlinear solvers. [37]

Naturally, in the reality, the concept of dissipated energy is more complicated than that described in this chapter. However, at this step of the research activity, a simplified approach has been proposed to reach a good compromise between accuracy and numerical efficiency because all the software parts of the HIL architecture (and especially the virtual train model comprising the degraded adhesion model) will have to be implemented in real-time. 


\subsection{The Controllers}

The controllers have to reproduce on the roller-rig the dynamical behaviour of the virtual railway vehicle under degraded adhesion conditions in terms of angular velocities $\omega_{w}$, applied torques $C_{s}$ and, consequently, tangential contact forces $T_{c}^{l / r}$. The inputs of the controller are the simulated tangential forces $T_{\text {sim }}$, the simulated wheelset angular velocities $\omega_{w s}$, the estimated wheel angular velocities $\widehat{\omega}_{w}$, the estimated motor torques $\hat{C}_{S}$ and the roller angular velocities $\omega_{r}^{l / r}$. The outputs are the 8 roller control torques $u^{l / r}$.

The controller layout consists of 8 independent controllers (one for each roller) and makes use of a sliding mode strategy based on the dynamical equations of the roller rig; this way, it is possible to reduce the disturbance effects due to the system nonlinearities and the parameter uncertainties [39] [40]. The total control torques $u^{l / r}$ are defined as:

$$
u^{l / r}=u_{\text {cont }}^{l / r}+u_{\text {disc }}^{l / r}+u_{\text {diff }}^{l / r}
$$

where the continuous control part $u_{\text {cont }}^{l / r}$ is built starting from the approximated 1D models of wheelset and rollers and by supposing negligible the slidings between the contact surfaces (on the roller-rig the adhesion conditions are good, with a friction coefficient $\mu_{\text {roll }}$ equal to 0.3$)$ :

$$
\begin{array}{cl}
C_{s}=J_{w} \dot{\omega}_{w}-T_{c}^{l} r_{w}-T_{c}^{r} r_{w} \\
u^{l}=J_{r} \dot{\omega}_{r}^{l}-T_{c}^{l} r_{w} \quad u^{r}=J_{r} \dot{\omega}_{r}^{r}-T_{c}^{r} r_{w} \\
\omega_{r}^{l}=-\frac{r_{w}}{r_{r}} \omega_{w} \quad \dot{\omega}_{r}^{l}=-\frac{r_{w}}{r_{r}} \dot{\omega}_{w} \\
\omega_{r}^{r}=-\frac{r_{w}}{r_{r}} \omega_{w} \quad \dot{\omega}_{r}^{r}=-\frac{r_{w}}{r_{r}} \dot{\omega}_{w}
\end{array}
$$

in which $r_{r}, r_{w}$ are the roller and wheelset radii and $J_{r}, J_{w}$ are their inertias. The choice of simplified 1D models has been necessary because the controllers are thought for a real-time implementation and, at the same time, the physical characteristics of the railway vehicle on the roller-rig are generally unknown. Consequenlty the models employed inside the estimators has to be as simple as possible.

By removing $T_{c}^{l}$ and $T_{c}^{r}$ in Eq. 15 , the following relation is obtained:

$$
C_{s}-\frac{r_{w}}{r_{r}}\left(u^{l}+u^{r}\right)=J_{t o t} \dot{\omega}_{w} \quad J_{t o t}=J_{w}+2\left(\frac{r_{w}}{r_{r}}\right)^{2} J_{r}
$$

where $J_{\text {tot }}$ is the total inertia of the rollers and the wheelset reduced to the wheelset rotation axis. Subsequently the desired wheelset dynamics is considered

$$
\hat{C}_{s}=J_{w} \dot{\omega}_{w s}+T_{s i m} r_{w}
$$

together with the sliding surface $S=\omega_{w s}-\omega_{w}=0$ and its time derivative $\dot{S}=$ $\dot{\omega}_{w s}-\dot{\omega}_{w}$ (since the sliding surface $S$ is not a physical surface, its physical dimensions depend on the dimensions of the control variables) [39] [40]. If the torque estimation $\hat{C}_{s} \simeq C_{s}$ is accurate enough, the sliding condition $\dot{S}=0$ can be obtained, starting from Eq. 16 and 17 , by taking

$$
\begin{gathered}
u=\frac{r_{r}}{r_{w}}\left[\left(1-\frac{J_{t o t}}{J_{s}}\right) \hat{C}_{s}+\frac{J_{t o t}}{J_{s}} T_{s i m} r_{w}\right] \\
u^{l}=\frac{u}{2} \quad u^{r}=\frac{u}{2} .
\end{gathered}
$$


On the other hand, $u_{d i s c}^{l / r}$ is the discontinuous control part related to the rejection of the disturbancies:

$$
u_{d i s c}^{l}=u_{d i s c}^{r}=k \Re\left(\omega_{w s}-\widehat{\omega}_{w}\right) .
$$

The discontinuous controls $u_{d i s c}^{l / r}$ are characterized by the gain $k$ and the function $\Re$ shown in Fig. 12 (the dead zone amplitude $\delta$ and the slope $\sigma$ are control parameters to be tuned).

Finally, $u_{d i f f}^{l / r}$ is an auxiliary control part aimed at synchronizing the roller angular

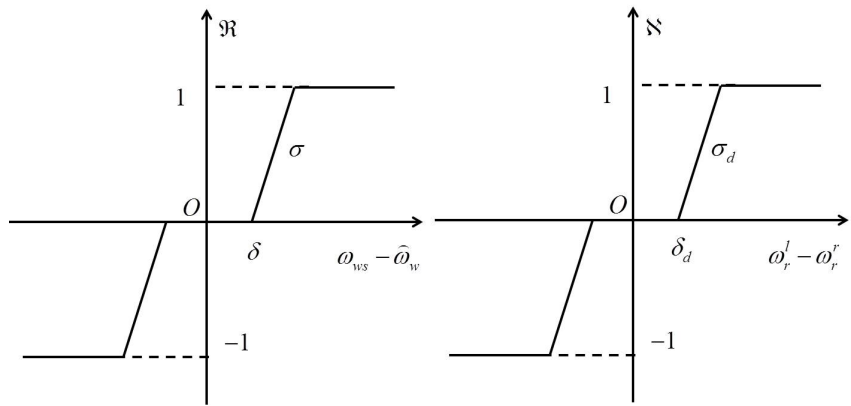

Figure 12: Discontinuous $\Re$ and auxiliary $\aleph$ control characteristics

velocities $\omega_{r}^{l}, \omega_{r}^{r}$ :

$$
u_{d i f f}^{l}=-k_{d} \aleph\left(\omega_{r}^{l}-\omega_{r}^{r}\right) \quad u_{d i f f}^{r}=k_{d} \aleph\left(\omega_{r}^{l}-\omega_{r}^{r}\right)
$$

The function $\aleph$ is reported in Fig. 12, while the parameters $k_{d}, \delta_{d}$ and $\sigma_{d}$ have to be tuned. The controller performances will be evaluated by means of the angular velocity error $e_{\omega}=\omega_{w s}-\omega_{w}$ and the torque estimation error $e_{c}=\hat{C}_{s}-C_{s}$. Limited values of the previuos errors $e_{\omega}, e_{c}$ assure a good estimation of the tangential contact forces $T_{c}^{l / r}$. According to the accuracy specifications of the project, reasonable tolerances are $1.0 \mathrm{rad} / \mathrm{s}$ for the angular velocity error $e_{\omega}$ and $1.0 * 10^{3} \mathrm{Nm}$ for the torque estimation error $e_{c}$.

\subsection{The Torque Estimators}

The estimators aim at evaluating the wheelset angular velocities $\widehat{\omega}_{w}$ and the torques applied to the wheelset $\widehat{C}_{s}$ starting from the roller angular velocities $\omega_{r}^{l / r}$ and the longitudinal reaction forces $T_{m i s}^{l / r}$ on the roller supports. Since the slidings between wheelset and rollers can be neglected, the following estimations approximately hold:

$$
\hat{\omega}_{w}=-\frac{r_{r}}{r_{w}} \frac{\omega_{r}^{l}+\omega_{r}^{r}}{2} \quad \hat{\dot{\omega}}_{w}=-\frac{r_{r}}{r_{w}} \frac{\dot{\omega}_{r}^{l}+\dot{\omega}_{r}^{r}}{2} \quad \hat{T}_{c}^{l / r}=T_{m i s}^{l / r} .
$$

Of course, the time derivative operation has to be robust, taking into account the numerical noise affecting $\omega_{r}^{l / r}$. To this end, a first-order discrete filter (i.e. a moving average filter with window size) has been used. The main features of this kind of 
discrete filter are the high numerical efficiency and the very low memory consumption. At this point, to estimate the motor torque applied to the wheelset, the estimator employes the simplified dynamical model of the wheelset:

$$
\hat{C}_{s}=J_{w} \hat{\dot{\omega}}_{w}-\hat{T}_{c}^{l} r_{w}-\hat{T}_{c}^{r} r_{w} .
$$

It is worth noting that, in this kind of applications, the estimators have to be necessarily simple because they are thought for a real-time implementation and, at the same time, the physical characteristics of the railway vehicle on the roller-rig are generally unknown. Consequenlty simplified 1D models of rollers and wheelsets have been employed inside the estimators.

\section{Experimental Data}

The HIL model performance have been validated by means of the comparison with the experimental data, provided by Trenitalia S. p. A. [46] and coming from on-track braking tests carried out in Velim (Czech Republic) with the coach UIC-Z1 [44]. The considered vehicle is equipped with a fully-working WSP system [45]. These experimental tests have been carried out on a straight railway track (over $2 \mathrm{~km}$ long) with good weather conditions. The wheel profile is the ORE S1002 (with a wheelset width $d_{w}$ equal to $1.5 \mathrm{~m}$ ) while the rail profile is the UIC60 (with a gauge $d_{r}$ equal to $1.435 \mathrm{~m}$ and a laying angle $\alpha_{p}$ equal to $1 / 20 \mathrm{rad}$ ).

The main characteristics of the braking test, considered as benchmark in this paper, are summarized in Tab. 6 (comprising the main wheel, rail and contact parameters; see chapter 3.2.2). [11] [12] [13] [14] [15] [16] [42]

The value of the kinetic friction coefficient under degraded adhesion conditions $\mu_{c d}$

Table 6: Main wheel, rail and contact parameters

\begin{tabular}{lcc}
\hline Parameter & Units & Value \\
\hline Initial train velocity $V$ & $\mathrm{~km} / \mathrm{h}$ & 120 \\
Nominal braking torque $C$ & $\mathrm{Nm}$ & 9500 \\
Measurement sample time $\Delta t_{s}$ & $\mathrm{~s}$ & 0.01 \\
Young modulus & {$[\mathrm{Pa}]$} & $2.1 * 10^{11}$ \\
Shear modulus & {$[\mathrm{Pa}]$} & $8.0 * 10^{10}$ \\
Poisson coefficient & {$[\mathrm{Ns} / \mathrm{m}]$} & 0.3 \\
Contact damping constant & - & $1.0 * 10^{5}$ \\
Polach reduction factor $k_{a d}$ & - & 0.3 \\
Polach reduction factor $k_{s d}$ & - & 0.1 \\
Polach reduction factor $k_{a r}$ & - & 1.0 \\
Polach reduction factor $k_{s r}$ & - & 0.4 \\
Kinetic friction coeffcient (degraded adhesion) $\mu_{c d}$ & - & 0.06 \\
Kinetic friction coeffcient (adhesion recovery) $\mu_{c r}$ & - & 0.28 \\
Friction ratio $A_{d}$ & - & 0.40 \\
Friction ratio $A_{r}$ & - & 0.40 \\
Friction decrease rate $\gamma_{d}$ & {$[\mathrm{~s} / \mathrm{m}]$} & 0.20 \\
Friction decrease rate $\gamma_{r}$ & {$[\mathrm{~s} / \mathrm{m}]$} & 0.60 \\
\hline
\end{tabular}

depends on the test performed on the track; the degraded adhesion conditions are usually reproduced using a watery solution containing surface-active agents, e.g. a solution sprinkled by a specially provided nozzle directly on the wheel-rail interface 
on the first wheelset in the running direction. The surface-active agent concentration in the solution varies according to the type of test and the desired friction level. The different degraded adhesion conditions have to be reproduced using a watery solution containing surface-active agents, e.g. a solution sprinkled by a specially provided nozzle directly on the wheel-rail interface on the first wheelset in the running direction (under a flow rate of $0,12 \mathrm{l} / \mathrm{min}$ to $0,19 \mathrm{l} / \mathrm{min}$ per tube through $8 \mathrm{~mm}$ diameter nozzles located along the longitudinal axis of the rail, a maximum of $70 \mathrm{~mm}$ from both the rail and the wheel).

The fluid used to reduce adhesion is an aqueous solution of a detergent with a fatty acid or surfactant base in a concentration below $15 \%$ and without mineral fillers. The detergent must be biodegradable, mix readily with water and be safe to dispose of in the track. The minimum concentration of the mixture must be such that for 1001 of water at least 11 of agent is employed.

The surface-active agent concentration in the solution varies according to the type of test and the desired friction level. Changing coefficients of adhesion are produced by switching the spraying device on and off intermittently.

Such procedure is standard in the railway filed (see the current regulation in force UNI EN 15595 [42]) and allows to obtain quite precise and uniform adherence levels on the rails along the line. In particular, it allows to reach the desired value of the kinematic friction coefficient $\mu_{c d}$ (see Tab. 6) under degraded adhesion conditions (without adhesion recovery) and, consequently, of the correspondent adhesion coefficient $f_{d}$.

On the other hand, the value of the kinetic friction coefficient under full adhesion recovery $\mu_{c r}$ corresponds to the classical kinetic friction coefficient under dry conditions.

During the experimental campaign, many different experimental tests have been performed (see [25] [24]). In this case for brevity and clarity reasons, a single test has been considered (a braking maneuver carried out on a straight track characterized by a uniform contaminant distribution constant along the line). In particular the experimental campaign showed that the vehicle velocity measurements are substantially repeatable. On the contrary, the sliding measurements are not repeatable due to complexity and chaoticity of the nonlinear system (characterized by discontinuities and threshold elements). To overcome this limitation, a statistical analysis is required.

Firstly the vehicle and wheelset velocities $v^{s p}, v_{w i}^{s p}=r_{w} \omega_{w i}^{s p}(i=1, \ldots, 4)$ are taken into account (see Fig. 13). Both the WSP intervention and the adhesion recovery in the second part of the braking maneuver are clearly visible.

Secondly the slidings among the wheelsets and the rails have been considered: $s_{i}^{s p}=v^{s p}-r_{w} \omega_{w i}^{s p}=v^{s p}-v_{w i}^{s p}$ (see Fig. 16, 17, 18 and 19). However these physical quantities cannot be locally compared to each other because of the complexity and the chaoticity of the system due, for instance, to the presence of discontinuous and threshold elements like the WSP. To better evaluate the behaviour of $s_{i}^{s p}$ from a global point of view, it is usefull to introduce the statistical means $\bar{s}_{i}^{s p}$ and the standard deviations 


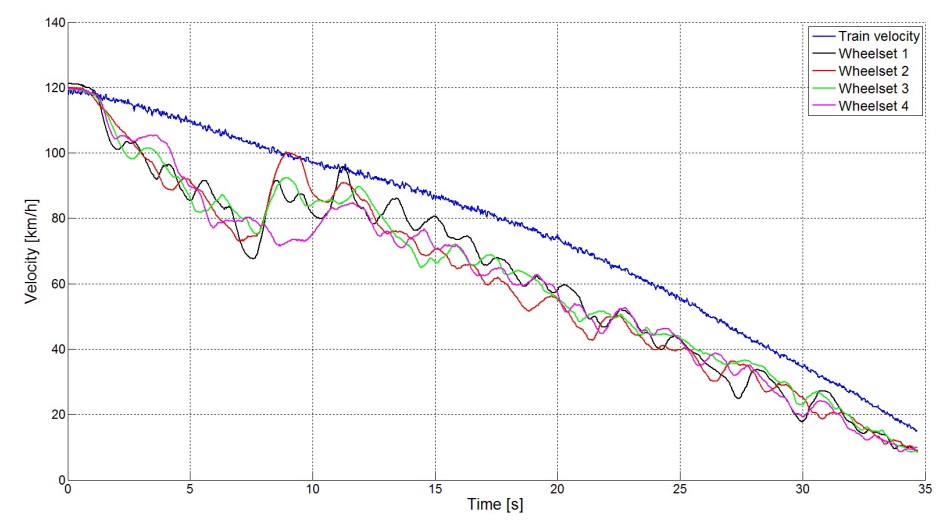

Figure 13: Experimental vehicle and wheelset velocities $v^{s p}, v_{w i}^{s p}=r_{w} \omega_{w i}^{s p}$

$\Delta_{i}^{s p}$ of the considered variables:

$$
\begin{gathered}
\bar{s}_{i}^{s p}=\frac{1}{T_{F}-T_{I}} \int_{T_{I}}^{T_{F}} s_{i}^{s p} d t \\
\Delta_{i}^{s p}=\sqrt{\frac{1}{T_{F}-T_{I}} \int_{T_{I}}^{T_{F}}\left(s_{i}^{s p}-\bar{s}_{i}^{s p}\right)^{2} d t}
\end{gathered}
$$

where $T_{I}$ and $T_{F}$ are respectively the initial and final times of the simulation (see Tab. 8).

The vehicle speed $v^{s p}$ is the longitudinal translation velocity of the railway vehicle while the wheel speed $v_{w i}^{s p}=r_{w} \omega_{w i}^{s p}$ is the longitudinal translation velocity of the wheel (proportional to the angular wheel velocity $\omega_{w i}^{s p}$ ). The two velocities are almost equal if almost pure rolling is present and differ in presence of sliding between wheel and rail. If the adhesion is degraded and the sliding is high, the impact on the vehicle speed is very important and causes the adhesion recovery due to the cleaning effect of the friction forces (and of the related dissipated energy) that destroy the contaminants. This way the increase of the available adherence leads, being equal the other system characteristics, to an increase (in modulus) of the vehicle deceleration.

\section{The Model Validation}

In this chapter the whole HIL architecture model is simulated and validated. More in detail, both the dynamical and the control performances of the system will be analyzed. The main control and integration parameters are summerized in Tab. 7)(see the paragraph 3.3] [38].

The control parameters $k, k_{d}, \delta, \delta_{d}, \sigma$ and $\sigma_{d}$ have been chosen taking in to account the performance of the actuators (IPM synchronous motors) and to obtain a good compromise among accuracy, stability and numerical stiffness. Analogously, also the numerical ODE solver has been selected to reach an effective compromise among precision, computational load and robustness against the model numerical stiffness. In particular, in this kind of applications, fixed step ODE solvers turn out to be more 
Table 7: Main control and integration parameters

\begin{tabular}{lcc}
\hline Parameter & Units & Value \\
\hline Control gain $k$ & $\mathrm{Nm}$ & $2 * 10^{4}$ \\
Dead zone amplitude $\delta$ & $\mathrm{rad} / \mathrm{s}$ & 0.05 \\
Control slope $\sigma$ & $\mathrm{Nms} / \mathrm{rad}$ & 10 \\
Control gain $k_{d}$ & $\mathrm{Nm}$ & $2 * 10^{4}$ \\
Dead zone amplitude $\delta_{d}$ & $\mathrm{rad} / \mathrm{s}$ & 0.05 \\
Control slope $\sigma_{d}$ & $\mathrm{Nms} / \mathrm{rad}$ & 10 \\
Integration algorithm & - & ODE5, Dormand-Prince \\
Algorithm characteristics & - & Fixed step, V order \\
Integration stepsize $\Delta t$ & $\mathrm{~s}$ & $10^{-4}$ \\
\hline
\end{tabular}

robust than the variable ones, providing, at the same time, the same accuracy and efficiency performances. Finally the choice of the ODE solver and its stepsize has to allow the real-time implementation of the virtual train model, the controllers and the torque estimators.

The simulated vehicle and wheelset velocities $v_{s}, v_{w s i}=r_{w} \omega_{w s i}$ are reported in Fig. 14). Figures 13 and 14 highlight a good qualitative matching between experimental and simulated data, both concerning the WSP intervention and the adhesion recovery in the second part of the braking maneuver.

The direct comparison between the experimental and simulated train velocities $v^{s p}$,

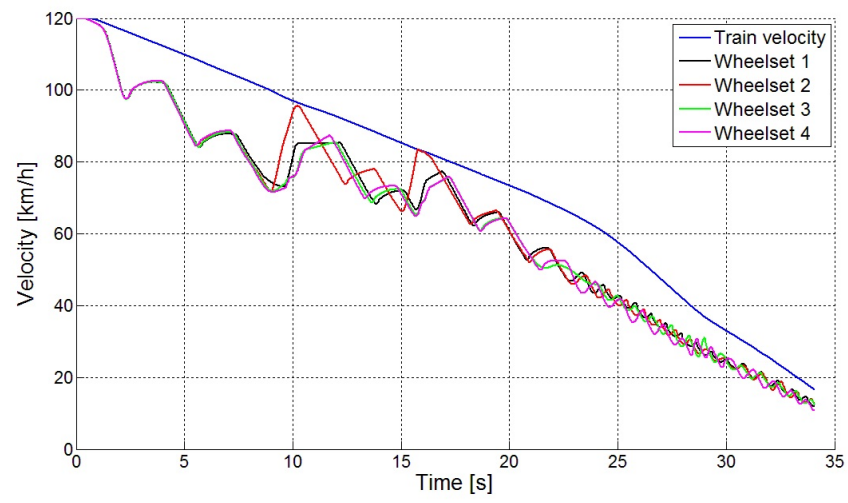

Figure 14: Simulated vehicle and wheelset velocities $v_{s}, v_{w s i}=r_{w} \omega_{w s i}$

$v_{s}$ is illustrated in Fig. 15 and shows also a good quantitative agreement between the considered quantities.

Subsequently, according to chapter 4, the simulated slidings among wheelsets and rails $s_{s i}=v_{s}-r_{w} \omega_{w s i}=v_{s}-v_{w s i}$ are taken into account and compared to the experimental ones $s_{i}^{s p}$ (see Fig. 16, 17, 18 and 19,.

The matching between experimental and simulated slidings is qualitatively good. However, since these physical quantities cannot be locally compared to each other because of the complexity and the chaoticity of the system, the statistical means $\bar{s}_{s i}$ and the standard deviations $\Delta_{s i}$ of the simulated slidings $s_{s i}$ are introduced (according to Eq. 23) to better evaluate the global behaviour of analyzed variables (some proper standard normality tests have been performed to verify the normality of the distribution). The comparison between experimental $\bar{s}_{i}^{s p}, \Delta_{i}^{s p}$ and simulated $\bar{s}_{s i}, \Delta_{s i}$ statistical 


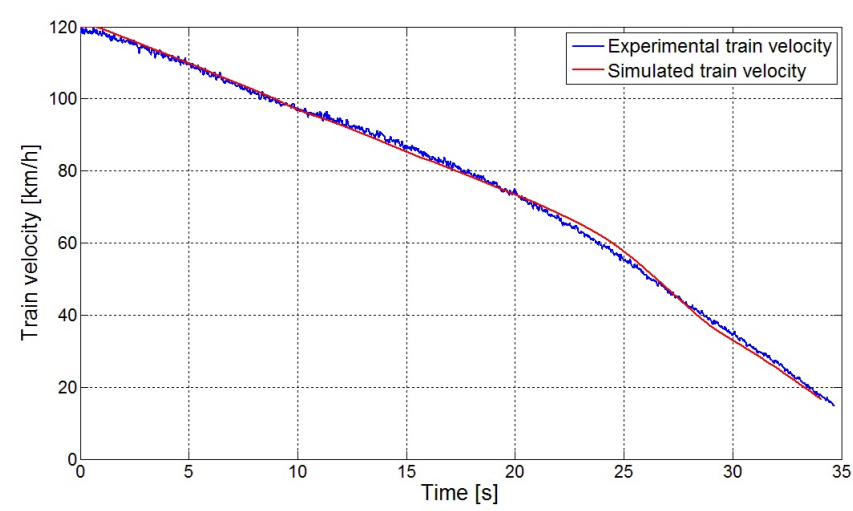

Figure 15: Experimental and simulated train velocities $v^{s p}, v_{s}$

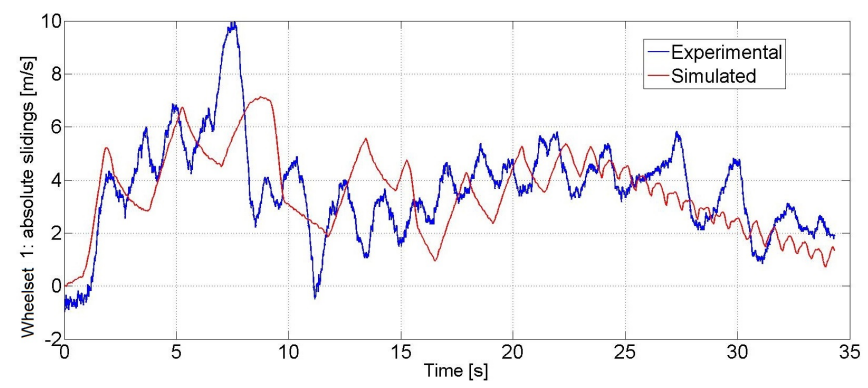

Figure 16: Experimental and simulated train slidings $s_{1}^{s p}, s_{s 1}$ for the first wheelset

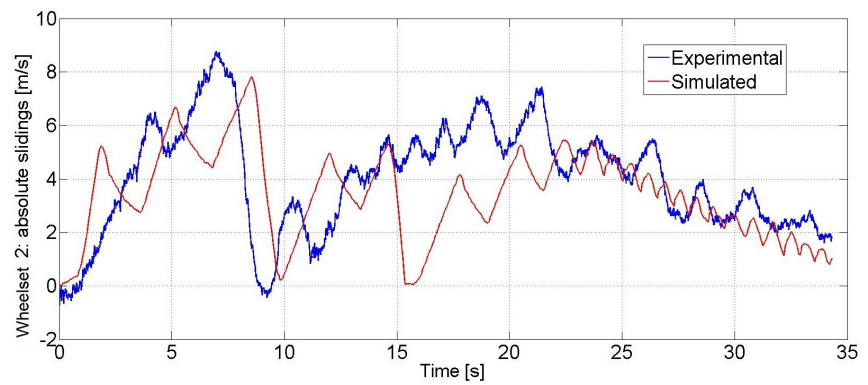

Figure 17: Experimental and simulated train slidings $s_{2}^{s p}, s_{s 2}$ for the second wheelset

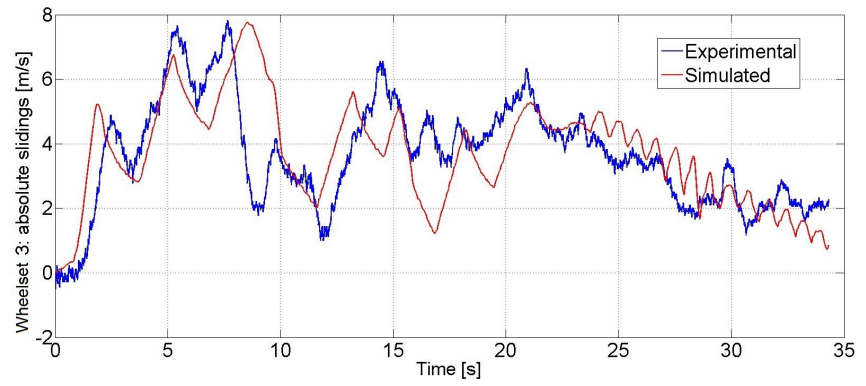

Figure 18: Experimental and simulated train slidings $s_{3}^{s p}, s_{s 3}$ for the third wheelset 


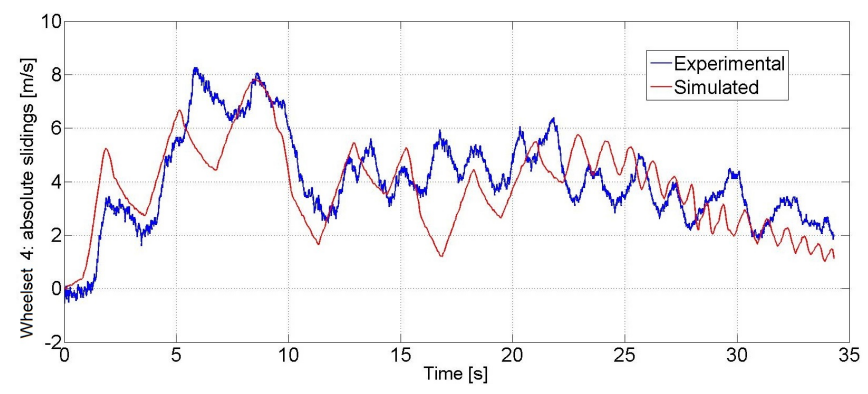

Figure 19: Experimental and simulated train slidings $s_{4}^{s p}, s_{s 4}$ for the fourth wheelset

indices is reported in Tab. 8 and highlights also a good quantitative match between the studied quantities.

The different frequency content and the possible time delay between experimen-

Table 8: Experimental $\bar{s}_{i}^{s p}, \Delta_{i}^{s p}$ and simulated $\bar{s}_{s i}, \Delta_{s i}$ statistical indices

\begin{tabular}{lllll}
\hline Wheelset & $\begin{array}{l}\bar{s}_{i}^{s p} \\
{[\mathrm{~km} / \mathrm{h}]}\end{array}$ & $\begin{array}{l}\bar{s}_{s i} \\
{[\mathrm{~km} / \mathrm{h}]}\end{array}$ & $\begin{array}{l}\Delta_{i}^{s p} \\
{[\mathrm{~km} / \mathrm{h}]}\end{array}$ & $\begin{array}{l}\Delta_{s i} \\
{[\mathrm{~km} / \mathrm{h}]}\end{array}$ \\
\hline Wheelset 1 & 13.08 & 12.82 & 6.45 & 6.56 \\
Wheelset 2 & 13.36 & 12.88 & 7.25 & 6.95 \\
Wheelset 3 & 13.09 & 13.32 & 5.92 & 5.70 \\
Wheelset 4 & 13.52 & 13.59 & 6.22 & 5.82 \\
\hline
\end{tabular}

tal and numerical results are mainly caused by the two following reasons: numerical noise and distortion due to the measurement chain (considered only for the wheelsets angular velocities) and unmodeled dynamics, affecting the system behavior especially at high frequencies.

The controller performances are evaluated in terms of angular velocity error $e_{\omega}=$ $\omega_{w s}-\omega_{w}$ and the torque estimation error $e_{c}=\hat{C}_{s}-C_{s}$. Small values of the errors $e_{\omega}$, $e_{c}$ assure a good estimation of the tangential contact forces $T_{c}^{l / r}$. The time history of the angular velocity error $e_{\omega}$ is plotted in Fig. 20 and shows the control capabilty of stabilising the sistem and rejecting the disturbancies produced by the initial transient and the adhesion recovery in the second phase of the braking maneuvre.

The second part of the braking maneuver is really important because it is characterized by the adhesion recovery. During this phase the mechanical stresses of the system and the disturbances are much higher than those in the first part of the maneuver (see the errors increase during the interval $25-30 s$ and then the following stabilization in the interval $30-35 s$ ).

The torque estimation error $e_{c}=\hat{C}_{s}-C_{s}$ and the real torques applied to the wheelset $C_{s}$ are reported respectively in Fig. 21 and 22. Also in this case the controlllers turn out to be effective in reproducing the real torques applied to the wheelsets of the vehicle.

Finally, the result analysis highlights the control capability of reproducing on the roller-rig a generic wheel-rail degraded adhesion pattern calculated by the reference virtual railway vehicle model (in terms of angular velocities $\omega_{w}$, applied torques on the wheelsets $C_{s}$ and, consequently, in terms of tangential efforts $T_{c}^{l / r}$ exchanged between 


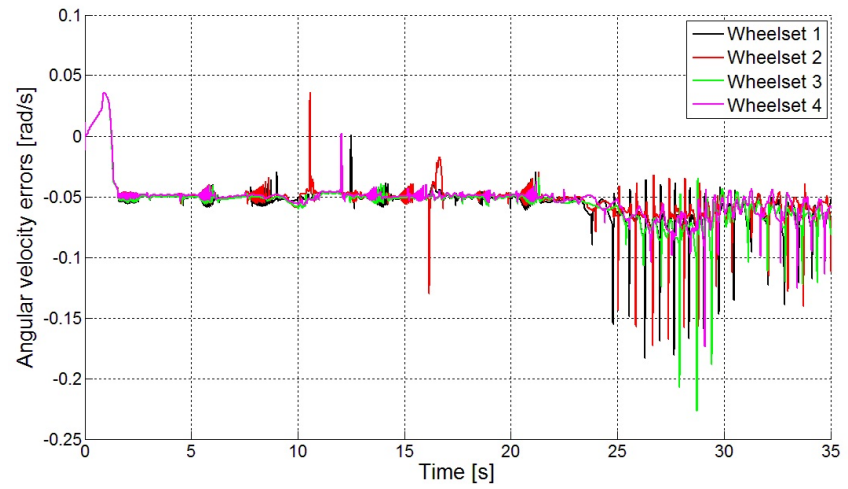

Figure 20: Angular velocity error $e_{\omega}=\omega_{w s}-\omega_{w}$

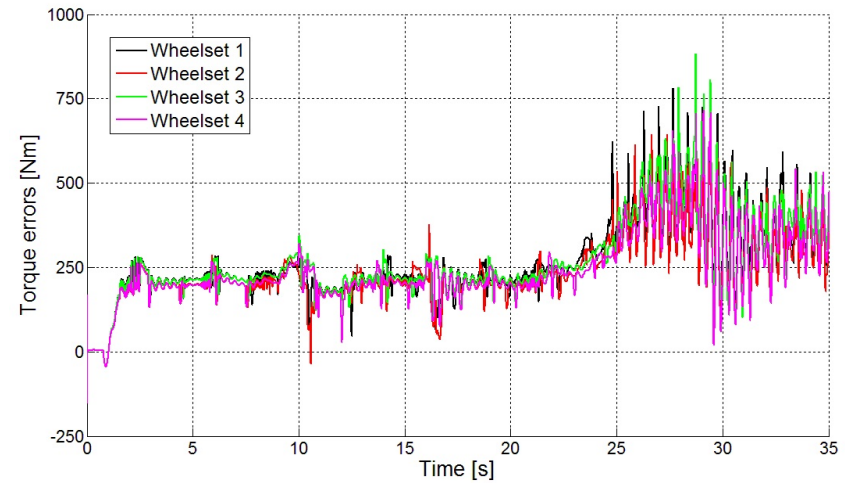

Figure 21: Torque estimation error $e_{c}=\hat{C}_{s}-C_{s}$

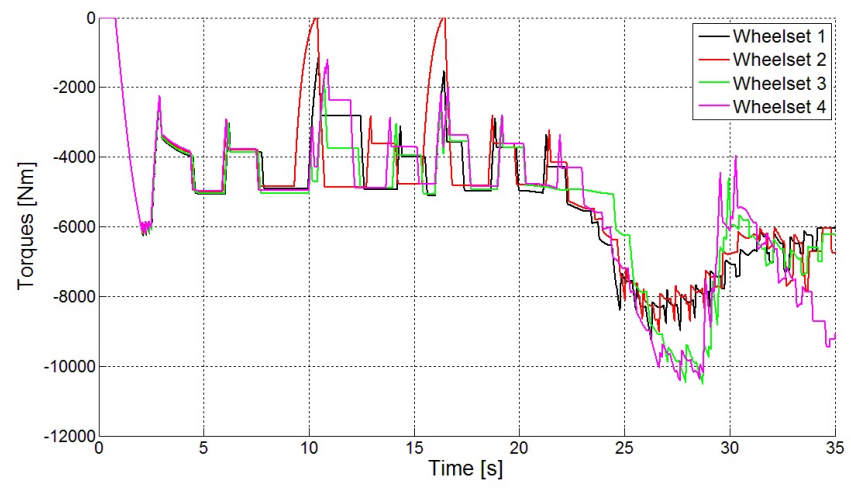

Figure 22: Torque applied to the wheelset $C_{s}$ 
the wheelsets and the rails).

\section{Conclusions}

In this work the authors described an innovative Hardware In the Loop (HIL) architecture to test braking on board subsystems on full-scale roller-rigs. The new strategy permits to reproduce on the roller-rig a generic wheel-rail adhesion pattern and, in particular, degraded adhesion conditions. The proposed approach has been preliminarily validated through on-track experimental data provided by Trenitalia and highlighted good performance in reproducing on the roller-rig the complex interaction between degraded adhesion conditions and railway vehicle dynamical behaviour during the braking manoeuvre.

From the railway industry point of view, the innovative full-scale roller-rig of the Firenze-Osmannoro research facility represents an important improvement if compared to the current state of the art. The main advantages of the new roller-rig concern the reduction of the expensive on-track tests (rent of vehicle and railway lines) and the possibility to test several on board subsystems on a roller-rig both with good and degraded adhesion conditions.

Currently, the construction of the test-rig is almost completed and the whole plant will be functioning very soon. Therefore, at this phase, the preliminary validation of the HIL architecture model performed comparing the whole model to suitable experimental data coming from on track tests has been very important because many parts of the simulated model will be part of the real HIL architecture (the virtual train model, the controllers and the estimators). During the next step of the research activity (scheduled for this winter), the HIL architecture model will be compared to experimental data directly coming from the real HIL architecture (comprising the real fully working test-rig) and considering scenarios characterized by adherence conditions variable along the track. This phase will be fundamental for the model validation and to draw solid conclusions about the test-rig effectiveness and efficiency.

From a theoretical point of view, more accurate adhesion models will be developed, taking into account new adhesion transition functions and considering wheel, rail and contaminants as elastic and fluid continuum bodies. Naturally these models will not able to be implemented in real-time but it will be employed as benchmark. In particular the real-time model, the continuum model and the experimental data (coming both from on track experimental campaigns and from the test-rig itself) will be compared to each other to better evaluate the benefits and the drawbacks of the proposed HIL architecture.

\section{Acknowledgements}

The authors wish to thank Trenitalia, SIMPRO and SICME Motors for supplying the technical and experimental data relative to the UIC-Z1 vehicle, the Firenze-Osmannoro 
roller-rig and the braking tests under degraded adhesion conditions.

\section{References}

[1] A. Jaschinki, H. Chollet, S. Iwnicki, "The application of the roller rigs to railway vehicle dynamics", Vehicle System Dynamics, vol. 31, pp. 325-344, 1999.

[2] R. Dukkipati, "A parametric study of the lateral stability of a railway bogie on a roller rig", Proceedings of the Institution of Mechanical Engineering Part F, vol. 213, pp. 39-47, 1999.

[3] K. Ahn, J. Park, S. Ryew, "The construction of a full-scale wheel/rail roller rig in Korea", Proceedings of the IEEE International Conference on Automation Science and Engineering (CASE) , pp. 802-803, 2012.

[4] N. Lee, C. Kang, W. Lee, T. Dongen, "Roller rig tests of a semi-active suspension system for a railway vehicle ", Proceedings of the IEEE International Conference on Control, Automation and Systems (ICCAS) , pp. 117-122, 2012.

[5] W. Zhang, J. Chen, X. Wu, X. Jin, "Wheel/rail adhesion and analysis by using full scale roller rig", Wear, vol. 253, pp. 82-88, 2002.

[6] M. Malvezzi, B. Allotta, L. Pugi, "Feasibility of degraded adhesion tests in a locomotive roller rig", Proceedings of the Institution of Mechanical Engineering Part F, vol. 222, pp. 27-43, 2008.

[7] R. Conti, E. Meli, L. Pugi, M. Malvezzi, F. Bartolini, B. Allotta, A. Rindi, P. Toni, "A numerical model of a HIL scaled roller rig for simulation of wheel-rail degraded adhesion condition", Vehicle System Dynamics, vol. 50, pp. 775-804, 2012.

[8] R. V. Dukkipati, "Lateral stability analysis of a railway truck on roller rig", Mechanism and Machine Theory, vol. 36, pp. 189-204, 2001.

[9] B. Allotta, R. Conti, M. Malvezzi, E. Meli, L. Pugi, A. Ridolfi, "Numerical simulation of a HIL full scale roller-rig model to reproduce degraded adhesion conditions in railway applications", Proceedings of the ECCOMAS 2012 Congress, 2012.

[10] J. J. Kalker, "Three-dimensional elastic bodies in rolling contact", Kluwer Academic Publishers, Norwell, MA, 1990.

[11] O. Polach, "Creep forces in simulations of traction vehicles running on adhesion limit”, Wear, vol. 258, pp. 992-1000, 2005.

[12] O. Polach, "A fast wheel-rail forces calculation computer code", Vehicle System Dynamics, vol. 33, pp. 728-739, 1999.

[13] J. Pombo, J. Ambrosio, "Application of a wheel-rail contact model to railway dynamics in small radius curved tracks", Multibody System Dynamics, vol. 19, pp. 91-114, 2008.

[14] J. Pombo, A. J. Silva, " A new wheel-rail contact model for railway dynamics", Vehicle System Dynamics, vol. 45, pp. 165-189, 2007.

[15] A. A. Shabana, K. E. Zaza, J. E. Escalona, J. R. Sany, ”Development of elastic force model for wheel/rail contact problems", Journal of Sound and Vibration, 
vol. 269, pp. 295-325, 2004.

[16] A. A. Shabana, M. Tobaa, H. Sugiyama, K. E. Zaazaa, "On the computer formulations of the wheel/rail contact problem", Nonlinear Dynamics, vol. 40, pp. 169-193, 2005.

[17] P. J. Blau, "Embedding wear models into friction models", Tribology Letters, vol. 34, pp. 75-79, 2009.

[18] M. Boiteux, "Le probleme de l'adherence en freinage", Revue generale des chemins de fer, pp. 59-72, 1986.

[19] P. Voltr, M. Lata, O. Cerny, "Measuring of wheel-rail adhesion characteristics at a test stand", Proceedings of XVIII Conference on Engineering Mechanics, 2012.

[20] A. T. Prez, G. G. Fatj, M. Hadfield, S. Austen, "A model of friction for a pin-ondisc configuration with imposed pin rotation", Mechanism and Machine Theory, vol. 46, pp. 1755-1772, 2011.

[21] M. Malvezzi, E. Meli, A. Rindi, S. Falomi, "Determination of wheel-rail contact points with semianalytic method", Multibody System Dynamics, vol. 20, pp. 327358, 2008.

[22] M. Malvezzi, E. Meli, S. Falomi, "Multibody modeling of railway vehicles: innovative algorithms for the detection of wheelrail contact points", Wear, vol. 271, pp. 453-461, 2011.

[23] S. Magheri, M. Malvezzi, E. Meli, S. Falomi, A. Rindi, "An innovative wheelrail contact model for multibody applications", Wear, vol. 271, pp. 462-471, 2011.

[24] B. Allotta, M. Malvezzi, E. Meli, L. Pugi, A. Ridolfi, A. Rindi, "Development of an innovative adhesion model for railway applications", Proceedings of the World Tribology Congress WTC 2013, 2013.

[25] B. Allotta, E. Meli, A. Ridolfi, A. Rindi, "Development of an innovative wheelrail contact model for the analysis of degraded adhesion in railway systems", Tribology International, vol. 69, pp. 128-140, 2013.

[26] E. Meli, A. Ridolfi, "An innovative wheelrail contact model for railway vehicles under degraded adhesion conditions", Multibody System Dynamics, doi:10.1007/s11044-013-9400-9, 2013.

[27] E. Meli, A. Ridolfi, A. Rindi, "An innovative degraded adhesion model for railway vehicles: development and experimental validation", Meccanica, doi:10.1007/s11012-013-9839-z, 2013.

[28] M. Ignesti, A. Innocenti, L. Marini, E. Meli, A. Rindi, "Development of a model for the simultaneous analysis of wheel and rail wear in railway systems", Multibody System dynamics, vol. 31, pp. 191-240, 2013.

[29] M. Ignesti, M. Malvezzi, L. Marini, E. Meli, A. Rindi, "Development of a wear model for the prediction of wheel and rail profile evolution in railway systems", Wear, vol. 284-285, pp. 1-17, 2012.

[30] J. Auciello, M. Ignesti, M. Malvezzi, E. Meli, A. Rindi, "Development and validation of a wear model for the analysis of the wheel profile evolution in railway vehicles", Vehicle System dynamics, vol. 50, pp. 1707-1734, 2012.

[31] M. Ignesti, A. Innocenti, L. Marini, E. Meli, A. Rindi, "Development of a wear model for the wheel profile optimisation on railway vehicles", Vehicle System 
dynamics, vol. 51, pp. 1363-1402, 2013.

[32] R. Krishnan,"Permanent magnet synchronous and brushless DC motor drives", CRC Press, Taylor and Francis group, 2010.

[33] A. Vagati, G. Pellegrino, P. Guglielmi, "Design tradeoff between constant power speed range, uncontrolled generator operation and rated current of IPM motor drives", IEEE Transactions on Industry Applications, vol. 47, pp. 1995-2003, 2011.

[34] S. Li, C. Xia, X. Zhou, "Disturbance rejection control method for permanent magnet synchronous motor speed-regulation system", Mechatronics, vol. 22, pp. 706-714, 2012.

[35] N. T. Vu, H. H. Choi, J. Jung, "Certainty equivalence adaptive speed controller for permanent magnet synchronous motor", Mechatronics, vol. 22, pp. 811-818, 2012.

[36] J. Nocedal, S. Wright, "Numerical optimization", Springer Series in Operation Research, Berlin, Germany, 1999.

[37] C. Kelley, "Iterative methods for linear and nonlinear equations", SIAM, Philadelphia, PA, 1995.

[38] L. Shampine, M. Reichelt, "The Matlab ODE suite", SIAM Journal of Scientific Computation, vol. 18, pp. 1-22, 1997.

[39] E. D. Sontag, "Mathematical Control Theory", Springer-Verlag, New York, 1998.

[40] H. K. Khalil, "Nonlinear Systems ", Prentice Hall, United States, 2002.

[41] F. Cheli, and E. Pennestri, "Cinematica e dinamica dei sistemi multibody", $C E A$, Milano, Italy, 2006.

[42] UNI-EN (2009) "Railway applications, Braking- Wheel Sliding Protection." UNI-EN 15595.

[43] "www.mathworks.com", Official Site of Mathworks, Natick, MA, 2013.

[44] Trenitalia SpA, "UIC-Z1 coach", Internal Report of Trenitalia, 2000.

[45] Trenitalia SpA, "WSP system”, Internal Report of Trenitalia, 2005.

[46] Trenitalia SpA, "On-track braking tests", Internal Report of Trenitalia, 2006.

[47] Trenitalia SpA, "Full-scale roller-rig: technical documentation", Internal Report of Trenitalia, 2011.

[48] SICME Motori, "IPM synchronous motor datasheet", Internal Report of SICME Motori, 2010. 